\title{
The stage-structured epidemic: linking disease and demography with a multi-state matrix approach model
}

\author{
Petra Klepac * $\quad$ Hal Caswell $^{\dagger}$
}

\begin{abstract}
Stage-structured epidemic models provide a way to connect the interacting processes of infection and demography. Reproduction and development can replenish the pool of susceptible hosts, and demographic structure leads to heterogeneous transmission and disease risk. Epidemics, in turn, can increase mortality or reduce fertility of the host population. Here we present a framework that integrates both demography and epidemiology in models for stage-structured epidemics. We use the vec-permutation matrix approach to classify individuals jointly by their demographic stage and infection status. We describe demographic and epidemic processes as alternating in time with a periodic matrix models. The application of matrix calculus to this framework allows for the calculation of $\mathscr{R}_{0}$ and sensitivity analysis.
\end{abstract}

KEYWORDS: disease dynamics; stage-structure; basic reproductive number; sensitivity

*Department of Ecology and Evolutionary Biology, Princeton, NJ 08544-1003, USA; pklepac@alum.mit.edu

${ }^{\dagger}$ Biology Department MS-34, Wood Hole Oceanographic Institution, Woods Hole, MA 02543, USA; Max Planck Institute for Demographic Research, Rostock, Germany 


\section{Introduction}

The basic epidemic models classify individuals on the basis of their infection status (susceptible, infected, recovered, immune, etc.), and determine the dynamics of the epidemic on the basis of the rates of individual movement among these infection states (Anderson \& May 1991). Individuals also and obviously differ in demographic characteristics (age, maturity, size, reproductive status, etc.). Demographic models describe the rates of individual movement among these stages, and the resulting population dynamics. These two kinds of movement are not independent; rates of survival, reproduction, and growth may be influenced by infection status, and rates of disease transmission, infection, and recovery may differ among demographic stages. Models for stage-classified epidemics provide a way to study the interaction between epidemic and demographic processes.

Stage-structured epidemic models face several challenges. They must classify individuals by two criteria, and keep track of the densities of all possible combinations of infection status and demographic stage. They must also recognise the frequent difference between demographic and epidemic time-scales. An individual that lives for many decades may experience many bouts of infection lasting a few weeks; sometimes disease outbreaks are highly seasonal and restricted to part of the year, while demographic processes may take place over many years.

There is a sizable literature on various aspects of the special case of age structure (e.g. Castillo-Chavez et al. 1989; Busenberg \& Hadeler 1990; Diekmann \& Heesterbeek 2000; Hethcote 2000; Dietz \& Heesterbeek 2002; Thieme 2003). However, especially in studies of plant and animal diseases, age may be inadequate as the state that characterises individuals (known as an $i$-state variable), and the infection process may depend more on physiological or behavioural stages than on age per se. Age, however, is a special case of stage, so the stage-structured epidemic includes age-classified as well as stage-classified models. 
Our stage-structured epidemic models classify individuals jointly by infection status and demographic stage, and include transitions on the short time scale of epidemic processes in a model in which demographic processes operate over longer time scales. The key to our formulation is the joint classification of individuals. We use the approach introduced by Hunter \& Caswell (2005); it was originally used to develop models classifying individuals by stage and spatial location, and has since been extended to consider stage and age, or stage and environmental states (Caswell 2009b). We combine this approach with the model for stage-structured disease transmission in Klepac et al. (2009).

Constructing a stage-classified epidemic model begins with a set of matrices that describe demographic processes, including the production of new individuals by reproduction and the roles (if any) of vertical transmission and inherited immunity. Then another set of matrices describe the infection dynamics, as a function of stage-specific transmission rates. Model development is facilitated by using the vec-permutation matrix to rearrange demographic stages and infection categories so that the matrices can be written in block diagonal form (Hunter \& Caswell 2005).

The result of this construction is a nonlinear matrix population model. Because demographic and epidemic processes alternate within a year, and do so repeatedly from year to year, the model is a periodic matrix model (Caswell 2001, Chapter 13). We will focus here on the asymptotic dynamics, particularly the possibility of endemic equilibrium, and on the calculation of the basic reproduction number $\mathscr{R}_{0}$. We will also use recent developments in perturbation analysis of nonlinear matrix population models to study the sensitivity of equilibria to changes in parameters.

Notation. We use lower case bold symbols (e.g., n) for vectors and upper case (N) for matrices. Our model will include many block-structured matrices, which 
we denote as, e.g., $\mathbb{N}$. Where convenient, we use MATLAB notation, where $N(i,:)$ and $N(:, j)$ refer to row $i$ and column $j$ of $\mathbf{N}$, respectively. We use subscripts when necessary to indicate the size of matrices or vectors; e.g., $\mathbf{I}_{s}$ is an identity matrix of order $s$. We use $\mathbf{1}_{s}$ to denote a $s \times 1$ vector of ones, and $\mathbf{e}_{j, s}$ to denote the $j$ th unit vector of length $s$ (i.e., column $j$ of $\mathbf{I}_{s}$ ). The transpose of $\mathbf{N}$ is denoted by $\mathbf{N}^{\top}$, the Kronecker product is $\otimes$, and the vec operator vec $(\mathbf{N})$ stacks the columns of a matrix one above the next.

\section{Model development}

We classify individuals into $s$ stages and $c$ infection states. As an example, we will develop the model for $s=2$ stages (juveniles and adults) and $c=3$ infection categories (susceptible, infected, and recovered), but the generalisation to more stages or more infection categories should be obvious.

The state of the population at time $t$ is given by the $s \times c$ array

$$
\mathbf{N}(t)=\left(\begin{array}{ccc}
n_{11} & \cdots & n_{1 c} \\
\vdots & & \vdots \\
n_{s 1} & \cdots & n_{s c}
\end{array}\right)
$$

The array $\mathbf{N}(t)$ can be transformed into a vector suitable for population projection in two ways (Hunter and Caswell 2005), using the vec operator,

$$
\begin{aligned}
& \mathbf{n}=\operatorname{vec}(\mathbf{N})=\left(\begin{array}{c}
N(:, 1) \\
\vdots \\
N(:, c)
\end{array}\right) \\
& \mathbf{m}=\operatorname{vec}\left(\mathbf{N}^{\top}\right)=\left(\begin{array}{c}
N(1,:)^{\top} \\
\vdots \\
N(s,:)^{\top}
\end{array}\right)
\end{aligned}
$$


The first of these stacks the columns of $\mathbf{N}$ one above the other, grouping stages together within infection categories. The second stacks the transposed rows of $\mathbf{N}$ one above the other, grouping infection categories within demographic stages.

At times during the annual cycle, the number of stages or infection states may change. In our example, reproduction will temporarily produce an extra stage of newborn individuals; the corresponding population vector has length $(s+1) c$ and we will denote it by $\mathbf{n}^{\prime}$.

The two population vectors are related by

$$
\operatorname{vec}\left(\mathbf{N}^{\top}\right)=\mathbf{K}_{s, c} \operatorname{vec}(\mathbf{N})
$$

where the matrix $\mathbf{K}_{s, c}$ is called the vec-permutation matrix (Henderson \& Searle, 1979,1981 ) or the commutation matrix (Magnus \& Neudecker, 1985). It is given by

$$
\mathbf{K}_{s, c}=\sum_{i=1}^{s} \sum_{j=1}^{c} \mathbf{E}_{i j} \otimes \mathbf{E}_{i j}^{\top}
$$

where $\mathbf{E}_{i j}$ is an $s \times c$ matrix with 1 in the $(i, j)$ position and zeros elsewhere, and $\otimes$ is the Kronecker matrix product. Since $\mathbf{K}$ is a permutation matrix, $\mathbf{K}^{\top}=\mathbf{K}^{-1}$.

\subsection{Demographic dynamics}

The population at any time-step consists of juveniles and adults that can either be susceptible, infectious, or recovered. Individuals in the stage $i$ and epidemic category $j$ suffer mortality $m_{i j}$ from natural, non-disease related causes. Juveniles in the epidemic category $i$ survive and grow to adults (in the same epidemic category) with the probability $g_{i}$. Adults in the epidemic category $i$ have the per-capita fertility $f_{i}$, and they produce newborns that are temporarily in three new demographic stages (see Figure 1 for an illustration). These offspring are distinguished from extant juveniles in order to account for infection status due to maternal immunity 
or vertical transmission. The demographic rates (survival, growth, reproduction) defined by Figure 1 are specified within infection category $i$ by a matrix $\mathbf{R}_{i}$, of dimension $((s+1) \times s)$

$$
\mathbf{R}_{i}=\left(\begin{array}{cc}
\left(1-g_{i}\right)\left(1-m_{1 i}\right) & 0 \\
g_{i}\left(1-m_{1 i}\right) & 1-m_{2 i} \\
0 & f_{i}
\end{array}\right) \quad i=1, \ldots, c
$$

In general, the first $s$ rows of the matrix $\mathbf{R}_{i}$ give the survival and transition of individuals among the $s$ demographic stages. The entries in row $s+1$ are the fertilities of each of the stages.

The demographic transitions for the whole population are given by

$$
\begin{aligned}
\mathbf{n}^{\prime}(t) & =\left(\begin{array}{l|l|l}
\mathbf{R}_{1} & & \\
\hline & \ddots & \\
\hline & & \mathbf{R}_{c}
\end{array}\right) \mathbf{n}(t) \\
& =\mathbb{R} \mathbf{n}(t)
\end{aligned}
$$

where $\mathbb{R}$ is a block-diagonal matrix containing the $\mathbf{R}_{i}$, and $\mathbf{n}^{\prime}$ is a vector of length $(s+1) c$.

\subsection{Parental effects}

The infection status of newborn individuals may be influenced by that of their parents, due to inherited immunity, vertical transmission of infection, or perhaps vaccination programs targeted at newborns. To account for these effects, and to allocate newborn offspring to the appropriate infection status, we define

$$
\begin{aligned}
& v=\text { proportion of offspring of infected parents that are infected } \\
& h=\text { proportion of offspring of recovered parents that are immune } \\
& \omega=\text { proportion of offspring of susceptible parents that are immune }
\end{aligned}
$$


In terms of these parameters, we define a matrix $\mathbf{G}$,

$$
\mathbf{G}=\left(\begin{array}{ccc}
1-\omega & 1-v & 1-h \\
0 & v & 0 \\
\omega & 0 & h
\end{array}\right)
$$

that allocates new offspring to the appropriate infection stages in the juvenile stage (Figure 2). We use $\mathbf{G}$ to update the population vector,

$$
\begin{aligned}
\mathbf{n} & =\left(\begin{array}{c|c|c}
\mathbf{I}_{c} & \mathbf{0} & \mathbf{G} \\
\hline \mathbf{0} & \mathbf{I}_{c} & \mathbf{0}
\end{array}\right) \mathbf{K}_{s+1, c} \mathbf{n}^{\prime} \\
& =\mathbb{M} \mathbf{K}_{s+1, c} \mathbf{n}^{\prime},
\end{aligned}
$$

where $\mathbb{M}=\left(\begin{array}{c|c|c}\mathbf{I}_{c} & \mathbf{0} & \mathbf{G} \\ \hline \mathbf{0} & \mathbf{I}_{c} & \mathbf{0}\end{array}\right)$

\subsection{Disease dynamics}

After accounting for survival, growth, and reproduction, we describe disease dynamics by a discrete-time SIR model specific to each demographic stage. By writing the population vector as $\mathbf{m}=\operatorname{vec}\left(\mathbf{N}^{\top}\right)$, we can use a block diagonal matrix to describe the dynamics,

$$
\begin{aligned}
\mathbf{m} & =\left(\begin{array}{l|l|l}
\mathbf{A}_{1}[\mathbf{m}] & & \\
\hline & \ddots & \\
\hline & & \mathbf{A}_{s}[\mathbf{m}]
\end{array}\right) \mathbf{m}(t) \\
& =\mathbb{A}[\mathbf{m}] \mathbf{m}
\end{aligned}
$$

The matrix $\mathbf{A}_{i}$ describes transitions among infection states

$$
\mathbf{A}_{i}=\left(\begin{array}{ccc}
1-\Lambda_{i}[\mathbf{m}] & 0 & 0 \\
\Lambda_{i}[\mathbf{m}] & 1-\gamma_{i} & 0 \\
0 & r_{i} \gamma_{i} & 1
\end{array}\right)
$$


In (14), $\Lambda_{i}[\mathbf{m}]$ is the probability of infection in stage $i$ (equivalent to instantaneous force of infection in continuous time models, or per capita rate at which susceptibles acquire infection) and depends on the entire vector $\mathbf{m}=\operatorname{vec}\left(\mathbf{N}^{\top}\right)$. The function $\Lambda[\cdot]$ depends on the type of transmission (e.g., Diekmann \& Heesterbeek 2000; Begon et al. 2002; Brauer 2006). We describe this dependence with a matrix $\boldsymbol{\beta}$ of transition rates (the 'who acquires infection from whom', or WAIFW matrix (Schenzle 1984; Anderson \& May 1985; Anderson \& May 1991; Dobson 2004)). The element $\beta_{i j}$ is the transmission rate from infected individuals in stage $j$ to susceptible individuals in stage $i$. The parameter $r_{i}$ is the probability of recovering from infection; $\left(1-r_{i}\right)$ is the probability of dying if infected.

For density-dependent transmission, usually appropriate for airborne diseases, the number of contacts is related to host density and the force of infection in stage $i$ depends on the total number of infectious individuals.

$$
\begin{aligned}
\Lambda_{i}(\mathbf{m}) & =1-\exp (-\beta(i,:) N(:, 2)) \\
& =1-\exp \left[-\mathbf{e}_{i, s}^{\top}\left(\boldsymbol{\beta} \otimes \mathbf{e}_{2, s}\right) \mathbf{m}\right]
\end{aligned}
$$

Frequency-dependent, or proportional mixing, transmission assumes a fixed number of contacts over the time-interval; and the infection probability for stage $i$ depends on the proportion of infectious cases in the total population,

$$
\Lambda_{i}(\mathbf{m})=1-\exp \left[\frac{-\mathbf{e}_{i, s}^{\top}\left(\boldsymbol{\beta} \otimes \mathbf{e}_{2, s}\right) \mathbf{m}}{\mathbf{1}_{s c}^{\top} \mathbf{m}}\right]
$$

where the denominator $\mathbf{1}_{s c}^{\top} \mathbf{m}$ is total population size.

\subsection{The stage-structured epidemic model}

To obtain a model for the stage-structured epidemic that includes all these processes, we form the periodic matrix product of $\mathbb{R}, \mathbb{M}$, and $\mathbb{A}$, using the vec- 
permutation matrix $\mathbf{K}$ to re-order the stages as necessary:

$$
\begin{aligned}
\mathbf{n}(t+1) & =\mathbf{K}_{s, c}^{\top} \mathbb{A}[\mathbf{m}] \mathbb{M} \mathbf{K}_{s+1, c} \mathbb{R} \mathbf{n}(t) \\
& =\mathbb{B}[\mathbf{n}(t)] \mathbf{n}(t)
\end{aligned}
$$

where $\mathbf{m}=\mathbb{M} \mathbf{K}_{s+1, c} \mathbb{R} \mathbf{n}(t)$ is the population just prior to the epidemic. Working from right to left, the model first accounts for demographic transitions and reproduction, then for parental effects on new offspring, and finally for transmission, infection, and recovery. The demographic dynamics include the effects of infection status (through the $\mathbf{R}_{i}$ ) and the disease dynamics include demographic effects (through the $\mathbf{A}_{i}$ ).

Note that the projection from $t$ to $t+1$ (say, from one year to the next) is described by a sequence of matrices on shorter, intra-annual time scale, and that the sequence is repeated to project from $t+1$ to $t+2$, and so on. Thus (18) is a periodic matrix model (Caswell 2001, Chapter 13). Such models can be analysed on both the interannual time scale, as in (19), or investigated on a finer scale using (18). The time-scale issue mentioned in the Introduction suggests that it may be desirable to change the ratio of demographic and epidemic transitions; we return to models with two time scales in Section 5.

\section{$3 \mathscr{R}_{0}$ for the stage-structured epidemic}

The occurrence of a disease outbreak depends on what happens upon introduction of an infection into an otherwise uninfected population. The reproductive number $\mathscr{R}_{0}$ gives the expected number of new infections produced by a single infected individual in a completely susceptible population (Dietz 1975; Diekmann et al. 1990; Anderson \& May 1991). If $\mathscr{R}_{0}>1$ the infection spreads and results in an epidemic; if $\mathscr{R}_{0}<1$ the infection dies out.

In the simplest models, $\mathscr{R}_{0}$ is the product of the rate of production of new 
infections and the average duration of the infectious state. When there are multiple infectious stages, $\mathscr{R}_{0}$ is the dominant eigenvalue of the next-generation matrix, given by $\mathbf{F}(\mathbf{I}-\mathbf{U})^{-1}$ where $\mathbf{F}$ is a matrix of rates of production of new infections and $\mathbf{U}$ is the matrix of transition rates among the infectious stages (cf. Cushing and Yicang 1994; van den Driessche \& Watmough 2002; Allen \& van den Driessche 2008).

In the stage-structured epidemic, which may include multiple infected classes and multiple demographic stages, $\mathscr{R}_{0}$ can be derived from an explicit invasion calculation.

The population dynamics are given by the nonlinear system (18). For ease of derivation, we write the susceptible, infected, and recovered components of $\mathbf{n}$ as

$$
\mathbf{n}=\left(\begin{array}{c}
\mathbf{n}_{S} \\
\mathbf{n}_{I} \\
\mathbf{n}_{R}
\end{array}\right)
$$

The attempted invasion of the infection takes place at a specified disease-free population

$$
\hat{\mathbf{n}}=\left(\begin{array}{c}
\hat{\mathbf{n}}_{S} \\
0 \\
0
\end{array}\right)
$$

Often, $\hat{\mathbf{n}}$ will be an equilibrium, but it could be some other population vector of interest. In a stage-structured epidemic, population size may be changing over time, and $\mathscr{R}_{0}$ may change with population size and structure.

To compute $\mathscr{R}_{0}$, we rewrite (18) as

$$
\left(\begin{array}{c}
\mathbf{n}_{S} \\
\mathbf{n}_{I} \\
\mathbf{n}_{R}
\end{array}\right)(t+1)=\left(\begin{array}{l|l|l} 
& & \\
\hline \mathbf{X}(\mathbf{n}) & \mathbf{Y}(\mathbf{n}) & \mathbf{Z}(\mathbf{n}) \\
\hline & &
\end{array}\right)\left(\begin{array}{c}
\mathbf{n}_{S} \\
\mathbf{n}_{I} \\
\mathbf{n}_{R}
\end{array}\right)(t),
$$

where the empty cells in the matrix contain expressions that are not used in this 
calculation. Following Allen \& van den Driessche (2008), we focus on the dynamics of $\mathbf{n}_{I}$, which are given by

$$
\mathbf{n}_{I}(t+1)=\mathbf{Y}[\mathbf{n}] \mathbf{n}_{I}(t)+\mathbf{X}[\mathbf{n}] \mathbf{n}_{S}+\mathbf{Z}[\mathbf{n}] \mathbf{n}_{R}
$$

The occurrence of an outbreak depends on the invasion exponent, which is the long-term growth rate of the linearisation of (23) near $\hat{\mathbf{n}}$, with $\mathbf{n}_{S}$ and $\mathbf{n}_{R}$ fixed at values $\hat{\mathbf{n}}_{S}$ and $\hat{\mathbf{n}}_{R}$, respectively. This linearization is given by

$$
\mathbf{J}=\left.\frac{d \mathbf{n}_{I}(t+1)}{d \mathbf{n}_{I}(t)}\right|_{\hat{\mathbf{n}}}
$$

(Verdy \& Caswell 2008). Note that the invasion of the infection could be determined directly from the dominant eigenvalue $\lambda$ of $\mathbf{J}$; the invasion will be successful if and only if $|\lambda|>1$. However, it is customary in epidemiology to study the invasion in terms of $\mathscr{R}_{0}$. See Wallinga \& Lipsitch (2007) for a relationship between $\lambda$ and $\mathscr{R}_{0}$. Differentiating (23) gives

$$
\begin{aligned}
d \mathbf{n}_{I}(t+1)= & (d \mathbf{Y}) \mathbf{n}_{I}(t)+\mathbf{Y} d \mathbf{n}_{I}(t)+(d \mathbf{X}) \hat{\mathbf{n}}_{S}(t)+(d \mathbf{Z}) \hat{\mathbf{n}}_{R}(t) \\
= & \left(\mathbf{n}_{I}^{\top}(t) \otimes \mathbf{I}\right) d \operatorname{vec} \mathbf{Y}+\mathbf{Y} d \mathbf{n}_{I}(t) \\
& +\left(\hat{\mathbf{n}}_{S}^{\top}(t) \otimes \mathbf{I}\right) d \operatorname{vec} \mathbf{X}+\left(\hat{\mathbf{n}}_{R}^{\top} \otimes \mathbf{I}\right) d \operatorname{vec} \mathbf{Z} .
\end{aligned}
$$

Evaluating at $\hat{\mathbf{n}}$ gives the Jacobian

$$
\mathbf{J}=\mathbf{Y}[\hat{\mathbf{n}}]+\left(\hat{\mathbf{n}}_{S} \otimes \mathbf{I}\right) \frac{d \operatorname{vec} \mathbf{X}}{d \mathbf{n}_{I}^{\top}}+\left(\hat{\mathbf{n}}_{R} \otimes \mathbf{I}\right) \frac{d \operatorname{vec} \mathbf{Z}}{d \mathbf{n}_{I}^{\top}}
$$

where all derivatives are evaluated at $\hat{\mathbf{n}}$.

The matrix derivatives $d \operatorname{vec} \mathbf{X} / d \mathbf{n}_{I}^{\top}$ and $d \operatorname{vec} \mathbf{Z} / d \mathbf{n}_{I}^{\top}$ are obtained from $\mathbb{B}$ using the same approach that we describe below in Section 4.1 to differentiate with respect to parameters.

To obtain the next-generation matrix, we must decompose $\mathbf{J}$ into transitions of extant infected individuals and the production of new infections.

$$
\mathbf{J}=\mathbf{F}+\mathbf{U}
$$


where $\mathbf{U}$ contains transitions of extant individuals and $\mathbf{F}$ the production of new individuals. Since infections reproduce themselves only by transmission, we can calculate $\mathbf{U}$ by setting transmission rates to zero and evaluating

$$
\mathbf{U}=\left.\mathbf{J}\right|_{\beta=0} .
$$

Then $\mathbf{F}=\mathbf{J}-\mathbf{U}$ and the reproductive number is

$$
\mathscr{R}_{0}=\max \operatorname{eig}\left(\mathbf{F}(\mathbf{I}-\mathbf{U})^{-1}\right) \text {. }
$$

\section{Dynamics of the stage-structured epidemic}

In the absence of the disease, the infection matrix $\mathbb{A}$ disappears from (18), leading to a linear model with projection matrix

$$
\mathbb{B}=\mathbf{K}_{s, c}^{\top} \mathbb{M} \mathbf{K}_{s+1, c} \mathbb{R}
$$

and the population will eventually grow exponentially at a rate given by the dominant eigenvalue of $\mathbb{B}$. Note that $\mathbb{B}$ may be reducible, because susceptible and recovered stages do not communicate in the absence of infection, but the eigenvalues of $\mathbb{B}$ still determine population growth. If the demography were densitydependent, then the disease-free dynamics would be nonlinear, and might include equilibria, cycles, invariant loops, or strange attractors. We do not consider these possibilities further.

The dynamics in the presence of the disease depend, in a complicated way, on the interaction between the infection dynamics and the effects of infection on the demographic rates. If the population grows to the point where $\mathscr{R}_{0}>1$, so that the disease can persist, and if infection reduces survival and reproduction sufficiently, our simulations show that it is possible for the disease to become endemic and regulate the population to a stable equilibrium. 
If an equilibrium exists on the annual time scale is a 2-cycle on the intra-annual time scale. The population oscillates between a state $\hat{\mathbf{n}}_{1}$ just before the epidemic and a state $\hat{\mathbf{n}}_{2}$ just after the epidemic. These vectors satisfy

$$
\begin{aligned}
& \hat{\mathbf{n}}_{1}=\mathbf{K}_{s, c}^{\top} \mathbb{M} \mathbf{K}_{s+1, c} \mathbb{R} \hat{\mathbf{n}}_{2} \\
& \hat{\mathbf{n}}_{2}=\mathbf{K}_{s, c}^{\top} \mathbb{A}\left[\mathbf{K}_{s, c} \hat{\mathbf{n}}_{1}\right] \mathbf{K}_{s, c} \hat{\mathbf{n}}_{1} .
\end{aligned}
$$

\subsection{Perturbation analysis of the endemic equilibrium}

The intra-annual cycle defined by $\hat{\mathbf{n}}_{1}$ and $\hat{\mathbf{n}}_{2}$ is determined by the parameters defining the demographic rates, the maternal influences, and the processes of disease transmission and recovery. Changes in these parameters will change $\hat{\mathbf{n}}_{1}$ and $\hat{\mathbf{n}}_{2}$. Perturbation analysis gives the sensitivity and elasticity of $\hat{\mathbf{n}}_{1}$ and $\hat{\mathbf{n}}_{2}$ to the parameters, which may be valuable in the design of public health interventions or in the interpretation of natural changes in parameters.

It is obvious from the construction of the stage-structured epidemic model that the effects of parameters on $\hat{\mathbf{n}}_{1}$ and $\hat{\mathbf{n}}_{2}$ can be very complicated. However, methods based on matrix calculus (Magnus \& Neudecker 1988) make the calculations possible. This approach has recently been applied in ecological contexts by Caswell (2006; 2007; 2008; 2009a; 2009b; Verdy \& Caswell 2008). In particular, we rely on methods given in Caswell (2008, Section 8). A short description of these methods is given in Appendix A.

Let $\boldsymbol{\theta}$ be a vector of parameters, of dimension $p \times 1$. Then the sensitivity of the equilibrium cycle is given by the derivatives of elements in $\hat{\mathbf{n}}_{1}$ and $\hat{\mathbf{n}}_{2}$ with respect to all parameters in $\boldsymbol{\theta}$; i.e., by

$$
\frac{d \hat{\mathbf{n}}_{i}}{d \boldsymbol{\theta}^{\top}} \quad i=1,2
$$

Each of these derivatives is a matrix, of dimension $s c \times p$, whose $(i, j)$ entry is the partial derivative of the $i$ th entry in $\hat{\mathbf{n}}_{i}$ with respect to the $j$ th entry of $\boldsymbol{\theta}$. 
To begin the calculation, we rewrite the 2-cycle in (31) and (32) as

$$
\begin{aligned}
& \hat{\mathbf{n}}_{1}=\mathbb{V} \hat{\mathbf{n}}_{2} \\
& \hat{\mathbf{n}}_{2}=\mathbb{W}\left[\hat{\mathbf{n}}_{1}\right] \hat{\mathbf{n}}_{1} .
\end{aligned}
$$

To find the sensitivity of the 2-cycle we differentiate (31) and (32), and apply the vec operator, the chain rule, and the first identification theorem (Magnus \& Neudecker 1985) to obtain

$$
\begin{aligned}
& \frac{d \hat{\mathbf{n}}_{1}}{d \boldsymbol{\theta}^{\top}}=\left(\hat{\mathbf{n}}_{2}^{\top} \otimes \mathbf{I}_{s c}\right) \frac{d \mathrm{vec} \mathbb{V}}{d \boldsymbol{\theta}^{\top}}+\mathbb{V} \frac{d \hat{\mathbf{n}}_{2}}{d \boldsymbol{\theta}^{\top}} \\
& \frac{d \hat{\mathbf{n}}_{2}}{d \boldsymbol{\theta}^{\top}}=\left(\hat{\mathbf{n}}_{1}^{\top} \otimes \mathbf{I}_{s c}\right) \frac{\partial \mathrm{vec} \mathbb{W}}{\partial \boldsymbol{\theta}^{\top}}+\left(\hat{\mathbf{n}}_{1}^{\top} \otimes \mathbf{I}_{s c}\right) \frac{\partial \mathrm{vec} \mathbb{W}}{\partial \hat{\mathbf{n}}_{1}^{\top}} \frac{d \hat{\mathbf{n}}_{1}}{d \boldsymbol{\theta}^{\top}}+\mathbb{W} \frac{d \hat{\mathbf{n}}_{1}}{d \boldsymbol{\theta}^{\top}}
\end{aligned}
$$

with all derivatives evaluated at $\left(\hat{\mathbf{n}}_{1}, \hat{\mathbf{n}}_{2}\right)$. The calculation of the derivatives of $\mathbb{V}$ and $\mathbb{W}$ is described in Appendix B.

We rewrite the system in block-matrix form as

$$
\begin{aligned}
\frac{d}{d \boldsymbol{\theta}^{\top}}\left(\begin{array}{c|c}
\hat{\mathbf{n}}_{1} \\
\hline \hat{\mathbf{n}}_{2}
\end{array}\right) & =\left(\begin{array}{c|c}
0 & \left(\hat{\mathbf{n}}_{2}^{\top} \otimes \mathbf{I}\right) \\
\hline\left(\hat{\mathbf{n}}_{1}^{\top} \otimes \mathbf{I}\right) & 0
\end{array}\right)\left(\begin{array}{c}
\frac{\partial \operatorname{vec} \mathbb{W}}{\partial \boldsymbol{\theta}^{\top}} \\
\frac{d \operatorname{vec} \mathbb{V}}{d \boldsymbol{\theta}^{\top}}
\end{array}\right) \\
& +\left[\left(\begin{array}{c|c|c}
0 & \left(\hat{\mathbf{n}}_{2}^{\top} \otimes \mathbf{I}\right) \\
\hline\left(\hat{\mathbf{n}}_{1}^{\top} \otimes \mathbf{I}\right) & 0
\end{array}\right)\left(\begin{array}{c|c|c}
0 & \frac{\partial \mathbb{W}}{\partial \hat{\mathbf{n}}_{1}^{\top}} \\
\hline 0 & 0
\end{array}\right)+\left(\begin{array}{c|c}
0 & \mathbb{V} \\
\hline \mathbb{W} & 0
\end{array}\right)\right] \frac{d}{d \boldsymbol{\theta}^{\top}}\left(\begin{array}{c}
\hat{\mathbf{n}}_{1} \\
\hline \hat{\mathbf{n}}_{2}
\end{array}\right) .
\end{aligned}
$$

To solve for the derivatives in (37), we define the following block matrices

$$
\begin{aligned}
& \mathbb{N}=\left(\begin{array}{l}
\hat{\mathbf{n}}_{1} \\
\hline \hat{\mathbf{n}}_{2}
\end{array}\right) \\
& \mathbb{F}=\left(\begin{array}{c|c}
0 & \mathbb{V} \\
\hline \mathbb{W}\left[\hat{\mathbf{n}}_{1}\right] & 0
\end{array}\right)
\end{aligned}
$$




$$
\begin{aligned}
\mathbb{H} & =\left(\begin{array}{c|c}
0 & \left(\hat{\mathbf{n}}_{2}^{\top} \otimes \mathbf{I}\right) \\
\hline\left(\hat{\mathbf{n}}_{1}^{\top} \otimes \mathbf{I}\right) & 0
\end{array}\right) \\
\mathbb{C} & =\left(\begin{array}{c|c}
0 & \frac{\partial \operatorname{vec} \mathbb{W}}{\partial \hat{\mathbf{n}}_{1}} \\
\hline 0 & 0
\end{array}\right) \\
\mathbb{D} & =\left(\begin{array}{c}
\frac{\partial \mathbb{W}\left[\hat{\mathbf{n}}_{1}\right]}{\partial \boldsymbol{\theta}^{\top}} \\
\frac{\partial \mathbb{V}}{\partial \boldsymbol{\theta}^{\top}}
\end{array}\right) .
\end{aligned}
$$

In terms of these matrices, (37) becomes

$$
\frac{d \mathbb{N}}{d \boldsymbol{\theta}^{\top}}=\mathbb{H} \mathbb{D}+[\mathbb{H} \mathbb{C}+\mathbb{F}] \frac{d \mathbb{N}}{d \boldsymbol{\theta}^{\top}}
$$

which can be solved for the sensitivities of $\hat{\mathbf{n}}_{1}$ and $\hat{\mathbf{n}}_{2}$ as in Caswell (2008), to give

$$
\frac{d \mathbb{N}}{d \boldsymbol{\theta}^{\top}}=\left(\mathbf{I}_{2 s c}-\mathbb{H} \mathbb{C}-\mathbb{F}\right)^{-1} \mathbb{H} \mathbb{D} .
$$

\subsection{Sensitivity of stage-specific prevalence}

The sensitivities of $\hat{\mathbf{n}}_{1}$ and $\hat{\mathbf{n}}_{2}$ given in (44) can be extended to examine the effects of parameters on stage-specific prevalence of the disease. At either of the $\hat{\mathbf{n}}_{i}$ (i.e., either just before or just after the epidemic), we can define the equilibrium prevalence as

$$
\hat{P}=\frac{\mathbf{a}^{\top} \hat{\mathbf{n}}}{\mathbf{b}^{\top} \hat{\mathbf{n}}}
$$

where $\mathbf{a}$ and $\mathbf{b}$ are vectors that depend on the classes being considered. The total prevalence $P_{\text {tot }}$ (total infected over total population size) is obtained by setting

$$
\begin{aligned}
\mathbf{a} & =\mathbf{e}_{2, c} \otimes \mathbf{1}_{s} \\
\mathbf{b} & =\mathbf{1}_{s c} .
\end{aligned}
$$


The stage-specific prevalence $\hat{P}_{j}$ (infected in stage $j$ over population in stage $j$ ) is obtained by setting

$$
\begin{aligned}
& \mathbf{a}=\mathbf{e}_{2, c} \otimes \mathbf{e}_{j, s} \\
& \mathbf{b}=\mathbf{1}_{c} \otimes \mathbf{e}_{j, s} .
\end{aligned}
$$

These prevalence measures are examples of ratios of weighted sums. In either case, the sensitivity of $\hat{P}$ is given by (Caswell 2008)

$$
\frac{d \hat{P}}{d \boldsymbol{\theta}^{\top}}=\left(\frac{\mathbf{b}^{\top} \hat{\mathbf{n}} \mathbf{a}^{\top}-\mathbf{a}^{\top} \hat{\mathbf{n}} \mathbf{b}^{\top}}{\left(\mathbf{b}^{\top} \hat{\mathbf{n}}\right)^{2}}\right) \frac{d \hat{\mathbf{n}}}{d \boldsymbol{\theta}^{\top}} .
$$

\subsection{Elasticities of the equilibrium and prevalence}

The calculation of calculation of elasticities, or proportional sensitivities, is described in Appendix A. The elasticities of the endemic equilibrium and equilibrium prevalence are given by

$$
\begin{aligned}
\frac{\varepsilon \mathbb{N}}{\varepsilon \boldsymbol{\theta}^{\top}} & =\operatorname{diag}(\mathbb{N})^{-1} \frac{d \mathbb{N}}{d \boldsymbol{\theta}^{\top}} \operatorname{diag}(\boldsymbol{\theta}) \\
\frac{\varepsilon \hat{P}}{\varepsilon \boldsymbol{\theta}^{\top}} & =\frac{1}{\hat{P}} \frac{d \hat{P}}{d \boldsymbol{\theta}^{\top}} \operatorname{diag}(\boldsymbol{\theta}) .
\end{aligned}
$$

\subsection{An example of the one time scale model}

As a numerical example of the stage-structured epidemic with one time scale (18), we develop a SIR-type model ( $c=3$ epidemic categories) with a single episode of disease transmission each year in a population with $s=2$ stages (juveniles and adults), for a potentially fatal disease with a long infectious period. We describe the contact process with two parameters; contact within stages $\left(\beta_{1}\right)$ and contact between juveniles and adults $\left(\beta_{2}\right)$, and we assume that $\left.\beta_{1}>\beta_{2}\right)$. This stage- 
Table 1: Parameters for the one and two time-scale models (subscripts $s, i, r$ denote infection states, $j, a$ denote demographic stages).

\begin{tabular}{l|l||l|l}
\multicolumn{2}{c||}{ One time scale model } & \multicolumn{2}{c}{ Two time scale model } \\
\hline parameter & value & parameter & value \\
\hline \hline$g_{s}=g_{r}$ & 0.4 & $g_{s}=g_{r}$ & 0.4 \\
$g_{i}$ & 0.3 & $g_{i}$ & 0.3 \\
$m_{j}^{(s)}=m_{j}^{(i)}=m_{j}^{(r)}$ & 0.1 & $m_{j}^{(s)}=m_{j}^{(i)}=m_{j}^{(r)}$ & 0.1 \\
$m_{a}^{(s)}=m_{a}^{(i)}=m_{a}^{(r)}$ & 0.2 & $m_{a}^{(s)}=m_{a}^{(i)}=m_{a}^{(r)}$ & 0.2 \\
$f_{s}=f_{r}$ & 0.3 & $f_{s}=f_{r}$ & 0.3 \\
$f_{i}$ & 0.2 & $f_{i}$ & 0.2 \\
$\beta_{11}=\beta_{22}$ & 0.0036 & $\frac{\beta_{11}}{k+1}=\frac{\beta_{22}}{k+1}$ & $3.564 \cdot 10^{-3}$ \\
$\beta_{12}=\beta_{21}$ & 0.0014 & $\frac{\beta_{12}}{k+1}=\frac{\beta_{21}}{k+1}$ & $1.386 \cdot 10^{-3}$ \\
$r_{j}$ & 0.6 & $r_{j}^{\left(\frac{1}{k+1}\right)}$ & 0.9950 \\
$r_{a}$ & 0.7 & $r_{a}^{\left(\frac{1}{k+1}\right)}$ & 0.9965 \\
$\gamma_{j}^{-1}=\gamma_{a}^{-1}$ & 10 & $\frac{\gamma}{k+1}$ & 0.099 \\
\end{tabular}


specific mixing pattern is captured with the WAIFW matrix,

$$
\boldsymbol{\beta}=\left(\begin{array}{ll}
\beta_{1} & \beta_{2} \\
\beta_{2} & \beta_{1}
\end{array}\right) .
$$

We assume density-dependent transmission (16). We assume that on average individuals stay infected for 10 years $\left(\gamma_{i}=1 / 10\right)$, after which they either recover with life-long immunity (with probability $r_{i}$ ), or die (with probability $1-r_{i}$ ).

The demographic part of the model includes all mortality not directly resulting from the disease. This mortality may depend on infection status, because infected individuals may be more susceptible to other diseases, or easier targets for predators. We assume no parental effects (maternal immunity, vertical transmission).

Parameter values for the single time scale model are given in Table 1, and the full matrices are given in Appendix C. With these parameters, the population grows exponentially in the absence of the disease at the rate $\lambda=1.023$. Infection introduces nonlinearity to the model that brings the population to an equilibrium. In this example $\mathscr{R}_{0}=2$, at an initial disease-free population of

$$
\mathbf{n}=\left(\begin{array}{llllll}
100 & 100 & 0 & 0 & 0 & 0
\end{array}\right)^{\top}
$$

and the convergence to the endemic equilibrium is shown in Figure 3. The equilibrium population is

$$
\mathbf{N}=\left(\begin{array}{ccc}
56.22 & 7.72 & 0.63 \\
63.94 & 33.31 & 11.20
\end{array}\right)
$$

The equilibrium prevalence in the entire population is $\hat{P}_{t o t}=0.24$. The stagespecific prevalences are $\hat{P}_{1}=0.12$ and $\hat{P}_{2}=0.31$.

The sensitivity of the equilibrium population just before the outbreak $\left(\hat{\mathbf{n}}_{1}\right)$ to the parameters, calculated according to (44), is shown in Figure 4a. An increase in transmission parameters (elements of $\boldsymbol{\beta}$ ) decreases the abundance of most stages 
due to increased outbreak intensity. Increase in juvenile transmission increases the level of juvenile infecteds but also of juvenile immune class through the process of recovery. Increases in stage-specific transmission lead to increased prevalence in that stage (Figure 4b). The importance of demographic parameters, especially the fertilities, on the equilibrium levels and prevalence becomes apparent when we look at the elasticities in Figure 5.

\section{A model with epidemic and demographic time scales}

In the stage-structured epidemic model (18), each year contains a single episode of demographic change and a single episode of epidemic change. However, many pathogens exhibit outbreaks on short time scales, on the order of days or weeks, rather than years. In such cases, a stage-structured epidemic model with a single time scale will either lose the details of the epidemic process, or describe the demography on an inappropriately short time scale. Our solution to this dilemma is to explicitly include two time scales in the model. ${ }^{1}$

To examine the infection process on a shorter time scale, we replace the matrix $\mathbb{A}[\mathbf{m}]$ in (18) with the product of a set of $k+1$ matrices

$$
\mathbb{A}[\mathbf{m}(t+k \Delta)] \cdots \mathbb{A}[\mathbf{m}(t+\Delta)] \mathbb{A}[\mathbf{m}(t)]
$$

where $\Delta<1$ is the time step on which the detailed trajectory of the outbreak is modelled. The resulting stage-structured epidemic is then

$$
\mathbf{n}(t+1)=\mathbf{K}_{s, c}^{\top}\{\mathbb{A}[\mathbf{m}(t+k \Delta)] \cdots \mathbb{A}[\mathbf{m}(t+\Delta)] \mathbb{A}[\mathbf{m}(t)]\} \mathbb{M} \mathbf{K}_{s+1, c} \mathbb{R} \mathbf{n}(t)
$$

\footnotetext{
${ }^{1}$ We note that our approach to the stage-structured epidemic has great flexibility in specifying time scales and seasonal variation. One could include multiple, alternating matrices corresponding to seasonal patterns of demographic and infection processes. The analysis of these models, using block matrices and the vec-permutation matrix, would follow the same sequence shown in the examples here.
} 
The disease transition matrices $\mathbb{A}[\cdot]$ depend on the population vector $\mathbf{m}$ at intermediate times between $t$ and $t+1$, given by

$$
\mathbf{m}(t+i \Delta)= \begin{cases}\mathbb{M} \mathbf{K}_{s+1, c} \mathbb{R} \mathbf{n}(t) & \text { if } i=0 \\ \mathbb{A}[\mathbf{m}(t+(i-1) \Delta)] \mathbf{m}(t+(i-1) \Delta) & \text { if } i=1, \ldots, k\end{cases}
$$

\subsection{An example of the two time-scale model}

In the model with two time scales, we scale the parameters so that the disease process gets the same amount of time as in the one time-scale example, but that time is divided up into finer intervals, to see more of the details of the infection process. This change in time scale changes the model from 1 to $k+1$ iterations of the epidemic transitions per population projection interval. Since we are now sampling the same time interval $k+1$ times, the duration of the infection, or $\gamma^{-1}$, now becomes $(k+1) \gamma^{-1}$, the survival probability in the finer interval is now $r^{\left(\frac{1}{k+1}\right)}$. The probability of escaping the infection during the $k+1$ steps is $(1-\Lambda)$, so the probability of escaping the infection during the one of the finer steps is $(1-$ $\Lambda)^{\left(\frac{1}{k+1}\right)}$ which requires $\boldsymbol{\beta} \rightarrow \frac{\boldsymbol{\beta}}{k+1}$.

To explore this model, we modified the example in Section 4.4 by including 101 epidemic iterations (i.e., $k=100$ ), and rescaled parameters for a two timescale model according to Table 1 . We set initial conditions to

$$
\mathbf{n}(0)=\left(\begin{array}{llllll}
100 & 100 & 1 & 0 & 0 & 0
\end{array}\right)^{\top} .
$$

Viewed on the annual time scale, the population converges to an equilibrium regulated by the disease (Figure 6). The equilibrium population is

$$
\hat{\mathbf{N}}=\left(\begin{array}{ccc}
60.84 & 18.29 & 3.15 \\
50.27 & 57.38 & 32.15
\end{array}\right)
$$

As expected the dynamics are qualitatively similar (but not identical, because of nonlinearitites) to those in Figure 3. On the intra-annual time scale, the oscillatory 
dynamics are apparent (Figure 6). The solution on the intra-annual time scale is a cycle of period $k+2$, satisfying

$$
\begin{gathered}
\hat{\mathbf{n}}_{1}=\mathbf{K}_{s, c}^{\top} \mathbb{M} \mathbf{K}_{s+1, c} \mathbb{R} \hat{\mathbf{n}}_{k+2} \\
\hat{\mathbf{n}}_{2}=\mathbf{K}_{s, c}^{\top} \mathbb{A}\left[\mathbf{K}_{s, c} \hat{\mathbf{n}}_{1}\right] \mathbf{K}_{s, c} \hat{\mathbf{n}}_{1} \\
\vdots \\
\hat{\mathbf{n}}_{k+2}=\mathbf{K}_{s, c}^{\top} \mathbb{A}\left[\mathbf{K}_{s, c} \hat{\mathbf{n}}_{k+1}\right] \mathbf{K}_{s, c} \hat{\mathbf{n}}_{k+1}
\end{gathered}
$$

The total prevalence at the annual equilibrium is $\hat{P}_{t o t}=0.34$. Stage specific prevalences are $\hat{P}_{1}=0.14$ and $\hat{P}_{2}=0.36$.

To calculate the sensitivity of the cycle, we rewrite (61) as

$$
\begin{aligned}
\hat{\mathbf{n}}_{1} & =\mathbb{V} \hat{\mathbf{n}}_{k+2} \\
\hat{\mathbf{n}}_{2} & =\mathbb{W}\left[\hat{\mathbf{n}}_{1}\right] \hat{\mathbf{n}}_{1} \\
& \vdots \\
\hat{\mathbf{n}}_{k+2} & =\mathbb{W}\left[\hat{\mathbf{n}}_{k+1}\right] \hat{\mathbf{n}}_{k+1}
\end{aligned}
$$

The sensitivity of this $k$-cycle is an extension of the analysis for the 2-cycle (see Caswell 2008, Section 8.1). Differentiating gives

$$
\begin{aligned}
\frac{d \hat{\mathbf{n}}_{1}}{d \boldsymbol{\theta}^{\top}} & =\quad\left(\hat{\mathbf{n}}_{k+2}^{\top} \otimes \mathbf{I}_{s c}\right) \frac{d \operatorname{vec} \mathbb{V}}{d \boldsymbol{\theta}^{\top}}+\mathbb{V} \frac{d \hat{\mathbf{n}}_{k+2}}{d \boldsymbol{\theta}^{\top}} \\
\frac{d \hat{\mathbf{n}}_{2}}{d \boldsymbol{\theta}^{\top}} & =\quad\left(\hat{\mathbf{n}}_{1}^{\top} \otimes \mathbf{I}_{s c}\right) \frac{\partial \operatorname{vec} \mathbb{W}\left[\hat{\mathbf{n}}_{1}\right]}{\partial \boldsymbol{\theta}^{\top}}+\left(\hat{\mathbf{n}}_{1}^{\top} \otimes \mathbf{I}_{s c}\right) \frac{\partial \operatorname{vec} \mathbb{W}\left[\hat{\mathbf{n}}_{1}\right]}{\partial \hat{\mathbf{n}}_{1}^{\top}}+\mathbb{W}\left[\hat{\mathbf{n}}_{1}\right] \frac{d \hat{\mathbf{n}}_{1}}{d \boldsymbol{\theta}^{\top}} \\
& \vdots \\
\frac{d \hat{\mathbf{n}}_{k+2}}{d \boldsymbol{\theta}^{\top}} & =\quad\left(\hat{\mathbf{n}}_{k+1}^{\top} \otimes \mathbf{I}_{s c}\right) \frac{\partial \operatorname{vec} \mathbb{W}\left[\hat{\mathbf{n}}_{k+1}\right]}{\partial \boldsymbol{\theta}^{\top}}+\left(\hat{\mathbf{n}}_{k+1}^{\top} \otimes \mathbf{I}_{s c}\right) \frac{\partial \mathrm{vec} \mathbb{W}\left[\hat{\mathbf{n}}_{k+1}\right]}{\partial \hat{\mathbf{n}}_{k+1}^{\top}}+\mathbb{W}\left[\hat{\mathbf{n}}_{k+1}\right] \frac{d \hat{\mathbf{n}}_{k+1}}{d \boldsymbol{\theta}^{\top}}
\end{aligned}
$$

with all matrices and derivatives evaluated at $\left(\hat{\mathbf{n}}_{1}, \ldots, \hat{\mathbf{n}}_{k+2}\right)$. To simplify and solve 
for the derivatives of the $\hat{\mathbf{n}}_{i}$, define the following block matrices:

$$
\mathbb{H}=\left(\begin{array}{c|c|c|c}
\mathbf{0} & & & \left(\hat{\mathbf{n}}_{k+2}^{\top} \otimes \mathbf{I}_{s c}\right) \\
\hline\left(\hat{\mathbf{n}}_{1}^{\top} \otimes \mathbf{I}_{s c}\right) & \mathbf{0} & & \\
\hline & \ddots & & \\
\hline \mathbf{0} & & \left(\hat{\mathbf{n}}_{k+1}^{\top} \otimes \mathbf{I}_{s c}\right) & \mathbf{0}
\end{array}\right)
$$

$\mathbb{D}=\left(\begin{array}{c}\frac{\partial \operatorname{vec} \mathbb{W}\left[\hat{\mathbf{n}}_{1}\right]}{\partial \boldsymbol{\theta}^{\top}} \\ \vdots \\ \frac{\partial \operatorname{vec} \mathbb{W}\left[\hat{\mathbf{n}}_{k+1}\right]}{\partial \boldsymbol{\theta}^{\top}} \\ \frac{\partial \mathrm{vec} \mathbb{V}}{\partial \boldsymbol{\theta}^{\top}}\end{array}\right)$

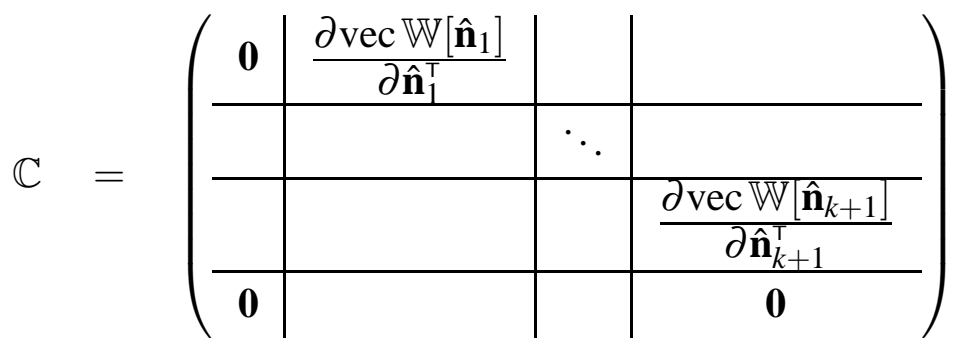

$\mathbb{F}=\left(\begin{array}{c|c|c|c}\mathbf{0} & \cdots & & \mathbb{V} \\ \hline \mathbb{W}\left[\hat{\mathbf{n}}_{1}\right] & & & \\ \hline & \ddots & & \vdots \\ \hline \mathbf{0} & & \mathbb{W}\left[\hat{\mathbf{n}}_{k+1}\right] & \mathbf{0}\end{array}\right)$

In terms of these matrices, and writing $\mathbb{N}=\left(\begin{array}{ccc}\hat{\mathbf{n}}_{1}^{\top} & \cdots & \hat{\mathbf{n}}_{k+2}^{\top}\end{array}\right)$, we have

$$
\frac{d \mathbb{N}}{d \boldsymbol{\theta}^{\top}}=\left(\mathbf{I}_{(k+2) s c}-\mathbb{H} \mathbb{C}-\mathbb{F}\right)^{-1} \mathbb{H} \mathbb{D}
$$

The derivatives of $\mathbb{V}$ and $\mathbb{W}$ are given in Appendix $B$. 
Figure 7 applies this analysis to show the sensitivity of $\hat{\mathbf{n}}_{1}$ (i.e., the equilibrium population immediately prior to the outbreak), and the corresponding elasticities are shown in Figure 8. The overall pattern of sensitivities is similar to that in the one time scale model (Figure 4). An increase in transmission parameters reduces the abundance of most stages, and increases in juvenile and adult transmission parameters lead to respective increases in juvenile and adult prevalence. Overall, sensitivities of $\hat{\mathbf{n}}_{1}$ in the two time-scale model are approximately two orders of magnitude greater than the same sensitivities in the single time-scale model. This reflects the fact that the epidemic processes operate 101 times during a single annual cycle, rather than once, and the corresponding scaling of parameters. The elasticities adjust for this rescaling - comparing Figures 5 and 8 reveals similar effects of proportional changes in demographic and epidemic parameters. Both in one time scale and the two time-scale example, the elasticities reveal the importance of demographic parameters, fecundities in particular, on the equilibrium stage abundances and the equilibrium prevalence.

\section{Discussion}

Any attempt to model the stage-structured epidemic must confront issues of multiple classification of individuals, multiple time scales, and nonlinearities. A stagestructured epidemic incorporates both demographic and epidemic processes, which interact with each other. The demographic stage of an individual may influence its susceptibility to disease, the mechanisms by which it transmits disease, and the mortality and morbidity that it experiences when infected. The infection status of an individual may influence its survival, growth, development, and reproduction, and thus the demographic processes leading to population growth or decline. This in turn affects the reproductive number. In at least some cases, the disease is able to regulate a population, that would otherwise grow exponentially, to a stable 
equilibrium $\hat{\mathbf{n}}$, with an endemic disease.

Stage-structured epidemics models are of interest in both human epidemiology and wildlife diseases. Demographic processes (births or immigration) replenish the pool of susceptibles. This can result in seasonal outbreaks that keep a pathogen circulating in the population. The effects of birth rate on epidemic dynamics have been observed in many natural systems. For example, changes in birth rates in individual states in the US have been shown to influence the spread of rota-virus in the US (Pitzer et al. 2009). High birth rates in Niger, in addition to strong seasonality, changes the dynamics of measles from the expected annual cycle to irregular, almost chaotic regime (Ferrari et al. 2008).

Population changes in plant and animal populations can be more extreme than those observed in human populations, and interactions of wildlife diseases with demographic and ecological processes are commonly reported (e.g., Härkönen et al. 2002; Lloyd-Smith et al. 2007; Klepac et al. 2009; Matser et al. 2009; Wasserberg et al. 2009). Wildlife populations are also more vulnerable to seasonal fluctuations and changes in environment (e.g., Altizer et al. 2006; Jenouvrier et al. 2009), which can lead to more devastating outbreaks of infectious diseases. Effects of the changing environment or seasonality can be studied in this framework by specifying disease-transmission matrices for different seasons.

Our approach to the stage-structured epidemic model classifies individuals jointly by their demographic stage and infection status, and describes demographic and epidemic processes as alternating in time. The respective transitions are described by simple block-structured matrices, a simplification made possible by the use of the vec-permutation matrix to rearrange the population vector. Multiple time scales are accommodated by the periodic matrix model format, in which the time step can vary within the overall projection interval. To summarise, the model construction proceeds as follows: 
1. Choose a set of demographic stages and infection states relevant to the question at hand and the time scales of interest.

2. For each infection state, develop a demographic matrix $\mathbf{R}$ from the fecundity and survival rates. Use these to create the block-diagonal matrix $\mathbb{R}$.

3. Based on information about parental effects, develop the block matrix $\mathbb{M}$ to assign new offspring to their infection states.

4. For each demographic stage, develop the epidemic matrix A appropriate to the choice of infection states. This step requires the choice of a disease transmission model and specification of the WAIFW matrix $\boldsymbol{\beta}$. Create the block-diagonal matrix $\mathbb{A}$ from the $\mathbf{A}_{i}$.

5. Based on the biology and epidemiology of the system decide on the ordering of the demographic and epidemic processes and the time scale for the epidemic interactions.

6. Construct the overall projection matrix $\mathbb{B}$ from (18) or (57).

The result of this construction is a nonlinear block-structured projection matrix $\mathbb{B}[\mathbf{n}]$. From this matrix one can calculate the linearization at a specified diseasefree state and the eigenvalues of the Jacobian matrix $\mathbf{J}$. These eigenvalues give the initial growth rate, or invasion exponent, for the disease, and determine whether an outbreak will occur at that disease-free state. If desired, one can also compute the next-generation matrix, and the reproductive number $\mathscr{R}_{0}$. Finally, our approach also gives the sensitivity analysis of $\hat{\mathbf{n}}$, and of functions calculated from $\hat{\mathbf{n}}$, such as stage-specific prevalence of the disease. These calculations are made possible by the application of matrix calculus to the periodic matrix model; the computations are straightforward in software (e.g., MATLAB, R, etc.) that is oriented to matrix manipulations.

The projection matrix $\mathbb{B}$ calculated from (18) or (57) is a large $(s \times c)$ blockstructured matrix whose entries are complicated (possibly very complicated) sums of products of mixtures of epidemic and demographic parameters. In principle, it 
would be possible to construct this matrix directly, by reasoning out all the possible transitions that an individual can make, and the parameters that determine those transitions. In practice, this is difficult and prone to error, which is one reason that the periodic matrix model formulation has proven useful in, e.g., spatial models (Hunter and Caswell 2005), variable environment models (Caswell 2006, 2009), and two-sex models (Jenouvrier et al. 2010).

Sensitivity and elasticity analysis can be applied in many ways to epidemic models. In addition to the sensitivity of a particular point in an epidemic cycle, one can, for example, compute the sensitivity of the average or variance over the cycle (Caswell 2008) to determine what influences the magnitude of an outbreak. Instead of focusing on $\mathscr{R}_{0}$ one could consider the initial growth rate of the epidemic and its sensitivity, to see what most affects the speed of disease invasion.

We have presented our approach in terms of an intentionally simple example, with two demographic stages and three infection states. However, we emphasise that it is not limited to this case. Our approach permits easy modification of either the demographic or the epidemic components. For example, the SIR model could be modified to include an exposed class with a latent period (SEIR model), or a class with waning immunity (SEIRS model). This would require only changing the matrices $\mathbf{A}_{i}$ that make up the block matrix $\mathbb{A}$. The construction of the projection matrix $\mathbb{B}$ automatically accounts for the new stages and the interaction of their rates with the other rates in the model, and the calculations of $\mathscr{R}_{0}$ and the sensitivity analysis of equilibrium follow directly from the same formulae. This ease in modifying the models could be useful in parameter estimation, by making it easier to compute likelihoods for a variety of different models structures.

Generalizations and future directions. Other than noting that our simulations show that endemic equilibria of populations regulated by disease effects are possible, we have not explored the full range of possible dynamics of the stage- 
structured epidemic. There are significant mathematical problems involving the existence of such equilibria, their local and global stability, and the possibility of other kinds of dynamics (cycles, invariant loops, strange attractors). If density-dependent demography is included, there can be complicated interactions between the demographic and epidemic nonlinearities. Our example used density-dependent disease transmission. Frequency-dependent transmission, or other models of contact and transmission processes, will change the patterns of dynamics.

Immunisation and treatment strategies can be incorporated into the model, on a seasonal and/or a stage-specific basis, by modifying parameters in the models given here (as in Metcalf et al (2010) for exploring measles vaccination strategies), or by incorporating immunisation matrices at the appropriate point in the annual cycle.

Parental effects (vertical transmission, inherited immunity) may be important in particular diseases. We have shown how to incorporate them, but their effects have yet to be studied in any detail.

We have focused on structure due to demographic stages. However, spatial structure can be treated as a special case of demographic stages, so our approach could be applied to spatially distributed epidemics of the sort studied by, e.g., Lloyd \& May (1996), Grenfell et al. (2001), Xia et al. (2004), and Viboud et al. (2006). In this case, matrices can be included that describe stage-specific migration rates; at any location, both immigration and births might affect the pool of susceptibles available for infection.

Acknowledgements The authors would like to thank Bryan Grenfell, Ottar Bjørnstad, Jessica Metcalf and Michael Neubert for helpful discussions, and three anonymous reviewers for detailed and valuable comments. P.K. acknowledges support of UNESCO-L'Oréal Fellowship "For Women in Science", Bill and Melinda 
Gates Foundation, National Institute of Health Grant R01-GM083983-01, National Science Foundation Grant 0742373. H.C. acknowledges support from National Science Foundation Grant DEB-0816514 and the Ocean Life Institute.

\section{A Appendix: Matrix calculus}

To calculate sensitivities in our approach we need to be able to take derivatives of matrices. Here we briefly review concepts from matrix calculus (Magnus and Neudecker 1988). The derivative of a vector $\mathbf{y}$ with respect to a vector $\mathbf{x}$ is a matrix whose $(i, j)$ entry is $\frac{d y_{i}}{d x_{j}}$. We denote the elasticities of $\mathbf{y}$ with respect to $\mathbf{x}$ as

$$
\frac{\varepsilon \mathbf{y}}{\varepsilon \mathbf{x}^{\top}}=\left(\frac{x_{j}}{y_{i}} \frac{d y_{i}}{d x_{j}}\right) .
$$

The elasticities are easily calculated from the sensitivities as

$$
\frac{\varepsilon \mathbf{y}}{\varepsilon \mathbf{x}^{\top}}=\operatorname{diag}(\mathbf{y})^{-1} \frac{d \mathbf{y}}{d \mathbf{x}^{\top}} \operatorname{diag}(\mathbf{x})
$$

(Caswell 2008) where $\operatorname{diag}(\mathbf{x})$ is a matrix with $\mathbf{x}$ on the diagonal and zeros elsewhere.

Vector derivatives are found by taking differentials and using the "first identification theorem" of Magnus and Neudecker (1988). The theorem states that if for some vectors $\mathbf{x}$ and $\mathbf{y}$ and some matrix $\mathbf{Q}$ it can be shown that

$$
d \mathbf{y}=\mathbf{Q} d \mathbf{x}
$$

then it holds that

$$
\frac{d \mathbf{y}}{d \mathbf{x}^{\top}}=\mathbf{Q}
$$

Derivatives of, or with respect to, matrices are obtained by transforming the matrices into vectors using the vec operator. Thus the derivative of $m \times n$ matrix 
$\mathbf{Y}$ and $p \times q$ matrix $\mathbf{X}$ is the $m n \times p q$ matrix

$$
\frac{d \operatorname{vec} \mathbf{Y}}{d \operatorname{vec}^{\top} \mathbf{X}}
$$

where $\operatorname{vec}^{\top} \mathbf{X}$ is short for $(\operatorname{vec} \mathbf{X})^{\top}$.

Using these rules, the chain rule holds - if $\mathbf{Y}$ is a function of $\mathbf{X}$ and $\mathbf{X}$ is a function of $\mathbf{Z}$, then

$$
\frac{d \operatorname{vec} \mathbf{Y}}{d \operatorname{vec}^{\top} \mathbf{Z}}=\frac{d \operatorname{vec} \mathbf{Y}}{d \operatorname{vec}^{\top} \mathbf{X}} \frac{d \operatorname{vec} \mathbf{X}}{d \operatorname{vec}^{\top} \mathbf{Z}}
$$

Finally, we also use theorem due to Roth (1934) that states that if

$$
\mathbf{Y}=\mathbf{A B C}
$$

it holds that

$$
\operatorname{vec} \mathbf{Y}=\left(\mathbf{C}^{\top} \otimes \mathbf{A}\right) \operatorname{vec} \mathbf{B}
$$

\section{B Appendix: Derivatives of block matrices}

The sensitivities of $\hat{\mathbf{n}}_{1}$ and $\hat{\mathbf{n}}_{2}$ require the derivatives of $\mathbb{V}$ and $\mathbb{W}$, which in turn require the derivatives of the block matrices $\mathbb{A}, \mathbb{M}$, and $\mathbb{R}$. In (37), the necessary derivatives of $\mathbb{V}$ and $\mathbb{W}$ are given by

$$
\begin{aligned}
& \frac{d \operatorname{vec} \mathbb{V}}{d \boldsymbol{\theta}^{\top}}=\left(\mathbb{R}^{\top} \mathbf{K}_{s+1, c}^{\top} \otimes \mathbf{K}_{s, c}^{\top}\right) \frac{d \operatorname{vec} \mathbb{M}}{d \boldsymbol{\theta}}+\left(\mathbf{I}_{s c} \otimes \mathbf{K}_{s, c}^{\top} \mathbb{M} \mathbf{K}_{s+1, c}\right) \frac{d \operatorname{vec} \mathbb{R}}{d \boldsymbol{\theta}^{\top}}(77) \\
& \frac{d \operatorname{vec} \mathbb{W}}{d \boldsymbol{\theta}^{\top}}=\left(\mathbf{K}_{s, c}^{\top} \otimes \mathbf{K}_{s, c}^{\top}\right) \frac{\partial \operatorname{vec} \mathbb{A}}{\partial \boldsymbol{\theta}^{\top}} \\
& \frac{d \operatorname{vec} \mathbb{W}}{d \mathbf{n}_{1}^{\top}}=\left(\mathbf{K}_{s, c}^{\top} \otimes \mathbf{K}_{s, c}^{\top}\right) \frac{\partial \operatorname{vec} \mathbb{A}}{\partial \mathbf{n}_{1}^{\top}} \mathbf{K}_{s, c}
\end{aligned}
$$

Because of the block structure of $\mathbb{R}, \mathbb{M}$, and $\mathbb{A}$ differentiation is of interest only with respect to the component matrices. These derivatives can be obtained (Caswell in prep.) as follows. 
We write

$$
\mathbb{A}=\sum_{i=1}^{s}\left(\mathbf{E}_{i i} \otimes \mathbf{A}_{i}\right)
$$

where $\mathbf{E}_{i i}$ is a $s \times s$ matrix with a 1 in the $(i, i)$ position and zeros elsewhere. Then it can be shown that

$$
\frac{d \operatorname{vec} \mathbb{A}}{d \boldsymbol{\theta}^{\top}}=\sum_{i=1}^{s}\left(\mathbf{I}_{c} \otimes \mathbf{K}_{s, c} \otimes \mathbf{I}_{s}\right)\left(\operatorname{vec} \mathbf{E}_{i i} \otimes \mathbf{I}_{c^{2}}\right) \frac{d \operatorname{vec} \mathbf{A}_{i}}{d \boldsymbol{\theta}^{\top}}
$$

(Caswell in prep.).

Similarly,

$$
\mathbb{R}=\sum_{i=1}^{c}\left(\mathbf{E}_{i i} \otimes \mathbf{R}_{i}\right)
$$

where now $\mathbf{E}_{i i}$ is a $c \times c$ matrix with a 1 in the $(i, i)$ entry and zeros elsewhere. Then

$$
\frac{d \operatorname{vec} \mathbb{R}}{d \boldsymbol{\theta}^{\top}}=\sum_{i=1}^{c}\left(\mathbf{I}_{c} \otimes \mathbf{K}_{s, c} \otimes \mathbf{I}_{s+1}\right)\left(\operatorname{vec} \mathbf{E}_{i i} \otimes \mathbf{I}_{s(s+1)}\right) \frac{d \operatorname{vec} \mathbf{R}_{i}}{d \boldsymbol{\theta}^{\top}}
$$

The block matrix $\mathbb{M}$ is not diagonal, but it can be written as

$$
\mathbb{M}=\left(\mathbf{E}_{11} \otimes \mathbf{I}_{c}\right)+\left(\mathbf{E}_{22} \otimes \mathbf{I}_{c}\right)+\left(\mathbf{E}_{13} \otimes \mathbf{G}\right)
$$

where in this case $\mathbf{E}_{i j}$ is a $s \times(s+1)$ matrix with a 1 in the $(i, j)$ entry and zeros elsewhere. The derivative of $\mathbb{M}$ is

$$
\frac{d \operatorname{vec} \mathbb{M}}{d \boldsymbol{\theta}^{\top}}=\left(\mathbf{I}_{s+1} \otimes \mathbf{K}_{s, c} \otimes \mathbf{I}_{c}\right)\left(\operatorname{vec} \mathbf{E}_{13} \otimes \mathbf{I}_{c^{2}}\right) \frac{d \operatorname{vec} \mathbf{G}}{d \boldsymbol{\theta}^{\top}} .
$$

\section{Appendix: Example matrices}

Transition matrices from the example in Section 4.4. $\mathbb{R}$ is $(9 \times 6)$ matrix with the following form for our parameter values: 


$$
\mathbb{R}=\left(\begin{array}{c|c|c}
\mathbf{R}_{1} & 0 & 0 \\
\hline 0 & \mathbf{R}_{2} & 0 \\
\hline 0 & 0 & \mathbf{R}_{3}
\end{array}\right)=\left(\begin{array}{cc|cc|cc}
0.54 & 0 & 0 & 0 & 0 & 0 \\
0.36 & 0.8 & 0 & 0 & 0 & 0 \\
0 & 0.3 & 0 & 0 & 0 & 0 \\
\hline 0 & 0 & 0.63 & 0 & 0 & 0 \\
0 & 0 & 0.27 & 0.8 & 0 & 0 \\
0 & 0 & 0 & 0.2 & 0 & 0 \\
\hline 0 & 0 & 0 & 0 & 0.54 & 0 \\
0 & 0 & 0 & 0 & 0.36 & 0.8 \\
0 & 0 & 0 & 0 & 0 & 0.3
\end{array}\right)
$$

In the example we ignore maternal immunity, vertical transmission, or vaccination at birth, so the matrix $\mathbb{M}$ that describes parental effects is simply:

$$
\mathbb{M}=\left(\begin{array}{lll|lll|lll}
1 & 0 & 0 & 1 & 0 & 0 & 0 & 0 & 0 \\
0 & 1 & 0 & 0 & 1 & 0 & 0 & 0 & 0 \\
0 & 0 & 1 & 0 & 0 & 1 & 0 & 0 & 0 \\
\hline 0 & 0 & 0 & 0 & 0 & 0 & 1 & 1 & 1 \\
0 & 0 & 0 & 0 & 0 & 0 & 0 & 0 & 0 \\
0 & 0 & 0 & 0 & 0 & 0 & 0 & 0 & 0
\end{array}\right)
$$

Epidemic transitions depend on the number of infected individuals at time $t$ :

$$
\begin{aligned}
\mathbb{A}[\mathbf{n}(t)] & =\left(\begin{array}{c|cc|ccc}
\mathbf{A}_{1}[\mathbf{n}(t)] & 0 \\
\hline 0 & \mathbf{A}_{2}[\mathbf{n}(t)]
\end{array}\right) \\
& =\left(\begin{array}{ccc|ccc}
e^{\left(-0.0036 n_{12}-0.0014 n_{22}\right)} & 0 & 0 & 0 & 0 & 0 \\
1-e^{\left(-0.0036 n_{12}-0.0014 n_{22}\right)} & 0.9 & 0 & 0 & 0 & 0 \\
0 & 0.06 & 1 & 0 & 0 & 0 \\
\hline 0 & 0 & 0 & e^{\left(-0.0014 n_{12}-0.0036 n_{22}\right)} & 0 & 0 \\
0 & 0 & 0 & 1-e^{\left(-0.0014 n_{12}-0.0036 n_{22}\right)} & 0.9 & 0 \\
0 & 0 & 0 & 0 & 0.07 & 1
\end{array}\right)
\end{aligned}
$$


The overall transition matrix $\mathbb{B}$ as defined in equation (19) and evaluated at initial conditions is

$$
\mathbb{B}=\left(\begin{array}{cccccc}
0.5394 & 0.2997 & 0 & 0.1998 & 0 & 0.2997 \\
0.0006 & 0.0003 & 0.6294 & 0.0002 & 0 & 0.0003 \\
0 & 0 & 0.0006 & 0 & 0.54 & 0 \\
0.3593 & 0.7984 & 0 & 0 & 0 & 0 \\
0.0007 & 0.0016 & 0.2697 & 0.7992 & 0 & 0 \\
0 & 0 & 0.0003 & 0.0008 & 0.36 & 0.8000
\end{array}\right)
$$




\section{References}

Allen LJ S, van den Driessche P (2008) The basic reproduction number in some discretetime epidemic models. J Differ Equ Appl 14:1127-1147

Altizer S, Dobson A, Hosseini P, Hudson P, Pascual M, Rohani, P (2006) Seasonality and the dynamics of infectious diseases. Ecol Lett 9:467-484 (doi:10.1111/j.14610248.2005.00879.x)

Anderson RM, May RM (1985) Age-related-changes in the rate of disease transmissions - implications for the design of vaccination programs. J Hyg-Cambridge 94:365-436

Anderson RM, May RM (1991) Infectious diseases of humans. Oxford: Oxford University Press

Begon M, Bennett M, Bowers RG, French NP, Hazel SM, Truner J (2002) A clarification of transmission terms in host-microparasite models: numbers, densities and areas. Epidemiol Infect 129:127-153

Brauer F (2006) Some simple epidemic models. Math Biosci Eng 3:1-15

Busenberg SN, Hadeler KP (1990) Demography and epidemics. Math Biosci 101:63-74

Castillo-Chavez C, Hethcote H, Andreasen V, Levin S, Liu W (1989) Epidemiological models with age structure, proportionate mixing, and cross-immunity. J Math Biol $27: 233-258$

Caswell H (2006) Applications of Markov chains in demography In: MAM2006: Markov Anniversary Meeting. Boson Books, Releigh, pp 319-334

Caswell H (2007) Evolutionary demography: the invasion exponent and the effective population density in nonlinear matrix models In: From energetics to ecosystems: the dynamics and structure of ecological systems. Springer, Dortrecht, pp 237-256

Caswell H (2008) Perturbation analysis of nonlinear matrix population models. Demographic Research 18: 59-116 
Caswell H (2009) $a$ Sensitivity and elasticity of density-dependent population models. J Differ Equ Appl 15:349-369 (doi:10.1080/10236190802282669)

Caswell H (2009)b Stage, age and individual stochasticity in demography. Oikos 118:1763-1782 (doi:10.1111/j.1600-0706.2009.17620.x)

Caswell H (in prep.) Sensitivity analysis of periodic matrix population models.

Cushing JM, Yicang Z (1994) The net reproductive value and stability in matrix population models. Natural Res Modeling 8:297-333

Diekmann O, Heesterbeek JAP (2000) Mathematical epidemiology of infectious diseases: model building, analysis and interpretation. John Wiley \& Son, LTD

Diekmann O, Hesterbeek JAP, Metz JAJ (1990) On the definition and the computation of the basic reproduction ratio $R_{0}$ in models for infectious diseases in heterogeneous populations. J Math Biol 28:365-382

Dietz K (1975) Transmission and control of arboviruses. In: Epidemiology Philadelphia: SIAM, pp 104-121

Dietz K, Heesterbeek JAP (2002) Daniel Bernoulli's epidemiological model revisited. Math Biosci 180:1-21

Dobson A (2004) Population dynamics of pathogens with multiple host species. Am Nat 164:S64-S78.

Ferrari MJ, Grais RF, Bharti N, Conlan AJK, Bjørnstad ON, Wolfson LJ, Guerin PJ, Djibo A, Grenfell BT (2008) The dynamics of measles in sub-Saharan Africa. Nature 451:679-684 (doi:10.1038/nature06509)

Grenfell BT, Bjørnstad ON, Kappey J (2001) Travelling waves and spatial hierarchies in measles epidemics. Nature 414:716-723

Härkönen T, Harding KC, Heide-Jørgensen MP (2002) Rates of increase in age-structured populations: a lesson from the European harbour seals. Can J Zool 80:1498-1510 
Henderson HV, Searle SR (1979) Vec and vech operators for matrices, with some uses in Jacobians and multivariate statistics. Can J Stat 7: 65-81

Henderson H, Searle S (1981) The vec-permutation, the vec operator and Kronecker products: a review. Linear Multilinear Algebra 9:271-288

Hethcote, H. 2000 The mathematics of infectious diseases. SIAM Rev., 42, 599-653

Hunter CM, Caswell H (2005) The use of the vec-permutation matrix in spatial matrix population models. Ecol Model 188: 15-21

Jenouvrier S, Caswell H, Barbraud C, Holland M, Stroeve J, Weimerskirch H (2009) Demographic models and IPCC climate projections predict the decline of an emperor penguin population. PNAS 106:1844-1847 (doi:10.1073/pnas.0806638106)

Jenouvrier S, Caswell H, Barbraud C, Weimerskirch H. (2010) Mating behavior, population growth and the operational sex ratio: a periodic two-sex model approach. Am Nat (in press)

Klepac P, Pomeroy LW, Bjørnstad ON, Kuiken T, Osterhaus AD, Rijks JM (2009) Stagestructured transmission of phocine distemper virus in the Dutch 2002 outbreak. Proc R Soc B 276: 2469-2476 (doi:10.1098/rspb.2009.0175)

Lloyd AL, May RM (1996) Spatial heterogeneity in epidemic models. J Theor Biol 179: $1-11$

Lloyd-Smith J, Greig D, Hietala S, Ghneim G, Palmer L, St Leger J, Grenfell B, Gulland F (2007) Cyclical changes in seroprevalence of leptospirosis in California sea lions: endemic and epidemic disease in one host species? BMC Infect Dis 7: 125 (doi: 10.1186/1471-2334-7-125)

Magnus J, Neudecker H (1985) Matrix differential calculus with applications to simple, Hadamard, and Kronecker products. J Math Psychol 29: 474-492

Magnus J, Neudecker H (1988) Matrix differential calculus with applications in statistics and econometrics. Wiley, New York 
Matser A, Hartemink N, Heesterbeek H, Galvani A, Davis S (2009) Elasticity analysis in epidemiology: an application to tick-borne infections. Ecol Lett 12:1298-1305 (doi: 10.1111/j.1461-0248.2009.01378.x)

Metcalf CJE, Klepac P, Ferrari M, Grais RF, Grenfell BT (2010) Modeling the first dose of measles vaccination: the role of maternal immunity, demographic factors, and delivery systems. Epidemiol Infect (in press)

Pitzer VE, Viboud C, Simonsen L, Steiner C, Panozzo CA, Alonso, WJ, Miller MA, Glass RI, Glasser JW et al. (2009) Demographic variability, vaccination, and the spatiotemporal dynamics of rotavirus epidemics. Science 325:290-294 (doi: $10.1126 /$ science.1172330)

Roth WE (1934) On direct product matrices. Bull Am Math Soc 40:461-468

Schenzle D (1984) An age-structured model of pre- and post-vaccination measles tranmission. Math Med Biol 1:169-191

Thieme HR (2003) Mathematics in population biology. Princeton Series in Theoretical and Computational Biology. Princeton Univeristy Press

van den Driessche P, Watmough J (2002) Reproduction numbers and subthreshold endemic equilibria for compartmental models of disease transmission. Math Biosci $180: 32-34$

Verdy A, Caswell H (2008) Sensitivity analysis of reactive ecological dynamics. Bull. Math. Biol. 70:1634-1659

Viboud C, Bjørnstad ON, Smith DL, Simonsen L, Miller MA, Grenfell BT (2006) Synchrony, waves and spatial hierarchy in the spread of influenza. Science 312:447-451

Wallinga J, Lipsitch M (2007) How generation intervals shape the relationship between growth rates and reproductive numbers. Proc R Soc B 274:599-604 
Wasserberg G, Osnas EE, Rolley RE, Samuel MD (2009) Host culling as an adaptive management tool for chronic wasting disease in white-tailed deer: a modelling study. J Appl Ecol 46:457-466 (doi:10.1111/j.1365-2664.2008.01576.x)

Xia Y, Bjørnstad ON, Grenfell BT (2004) Measles metapopulation dynamics: A gravity model for epidemiological coupling and dynamics. Am Nat 164:267-281 


\section{Figures}

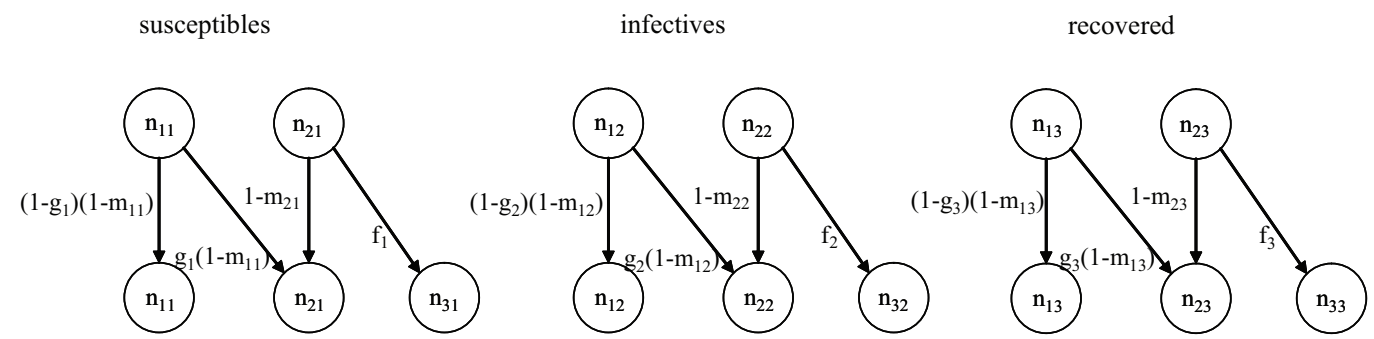

Figure 1: Reproduction matrix $\mathbb{R}$ accounts for the reproduction, survival and growth. New individuals temporarily show up in extra states $\left(\mathbf{n}_{3}\right.$.) before they are assigned to their epidemic categories. 

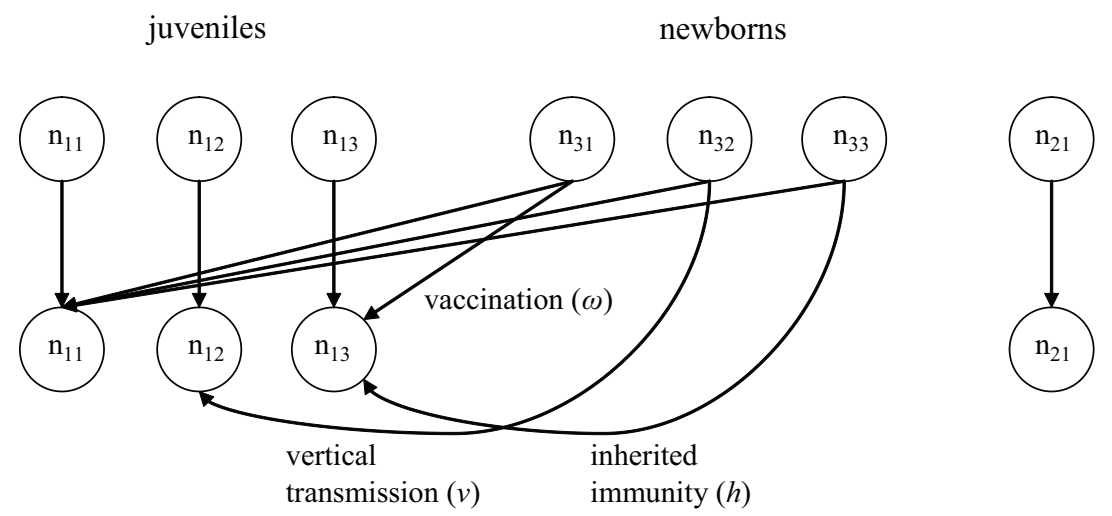

adults

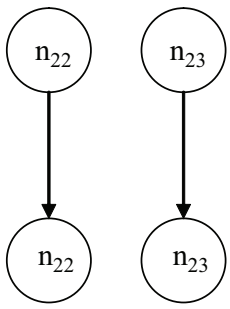

Figure 2: After reproduction, newborn individuals are assigned to their epidemic categories by the matrix $\mathbb{M}$. If there is no vertical transmission, inherited immunity or vaccination at birth $(\omega=0, h=0, v=0)$ then all newborn individuals will be susceptible. 


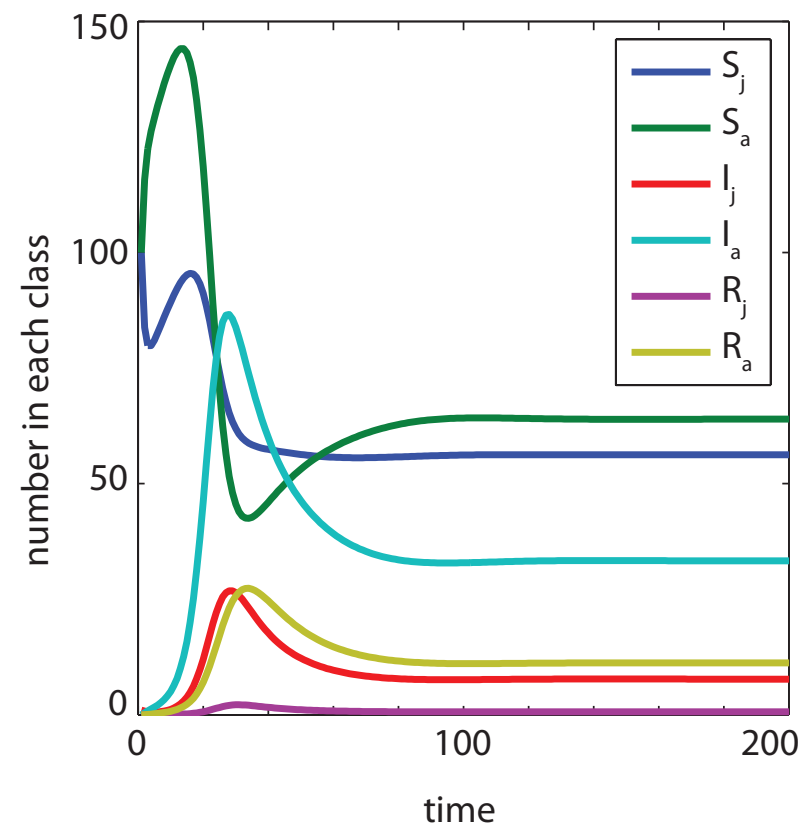

Figure 3: Endemic equilibrium of a stage-structured SIR model. Parameters in Table 1. 
(a)
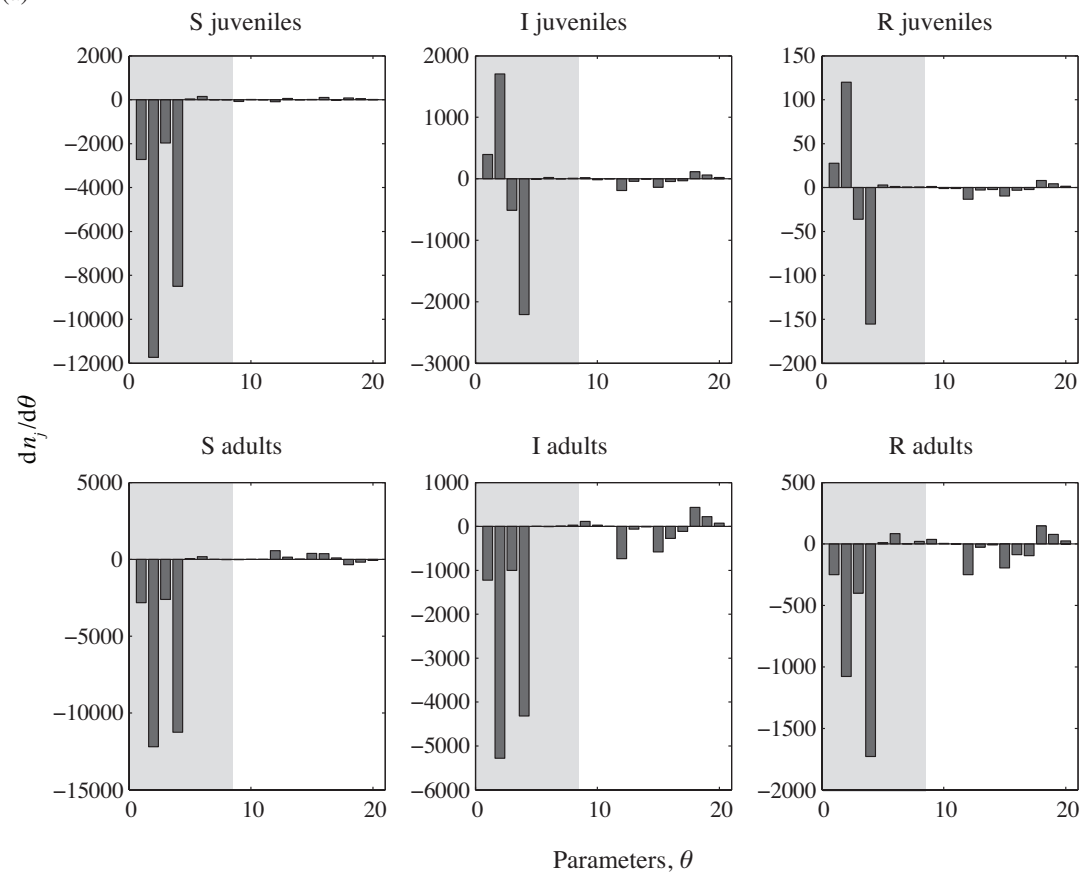

(b)
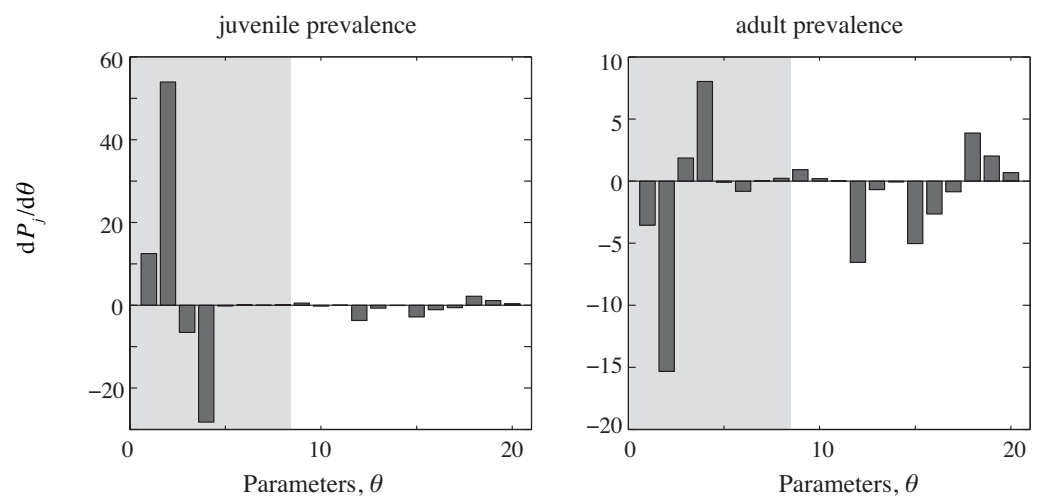

Figure 4: Stage-structured SIR model: (a) sensitivity of the equilibrium population just before the outbreak $\left(\hat{\mathbf{n}}_{1}\right)$; (b) sensitivity of disease prevalence. Sensitivities are shown to epidemic parameters in shaded region $\left(\begin{array}{llllllll}\beta_{11} & \beta_{21} & \beta_{12} & \beta_{22} & \gamma_{j} & \gamma_{a} & r_{j} & r_{a}\end{array}\right)$ and demographic parameters $\left(\begin{array}{llllllllllll}g_{s} & g_{i} & g_{r} & m_{j}^{(s)} & m_{j}^{(i)} & m_{j}^{(r)} & m_{a}^{(s)} & m_{a}^{(i)} & m_{a}^{(r)} & f_{s} & f_{i} & f_{r}\end{array}\right)$. See Table 1 for definitions and values. 
(a)
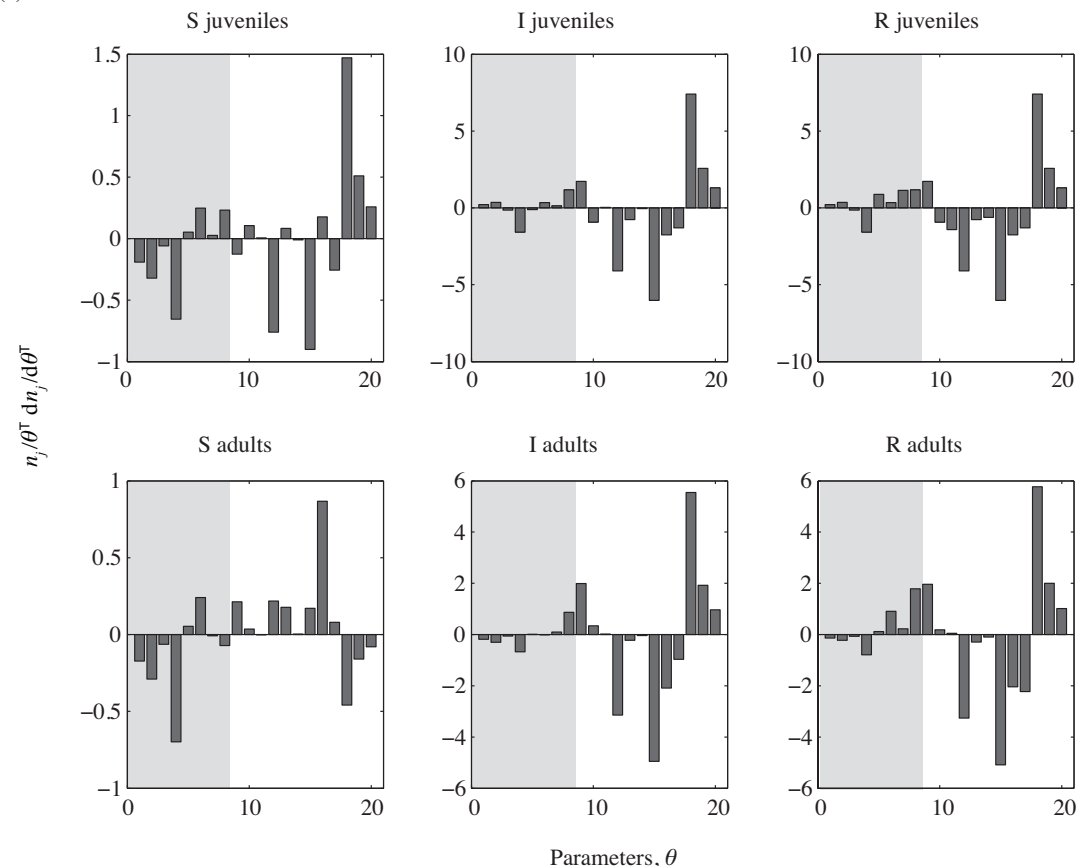

(b)
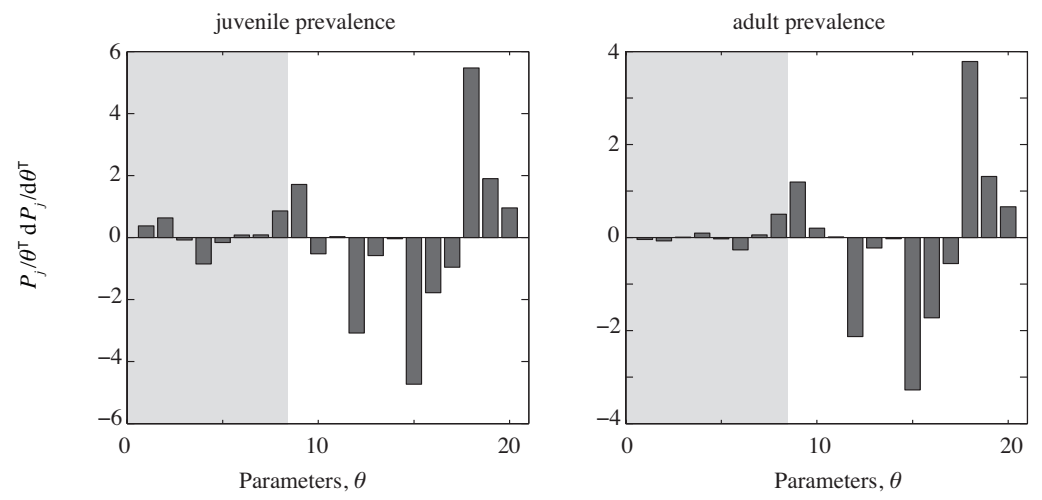

Figure 5: Stage-structured epidemic model with two time scales: (a) elasticities of the equilibrium population just before the outbreak $\left(\hat{\mathbf{n}}_{1}\right)$; (b) elasticity of disease prevalence. Parameters as in Figure 4; shaded regions correspond to the epidemic parameters. 

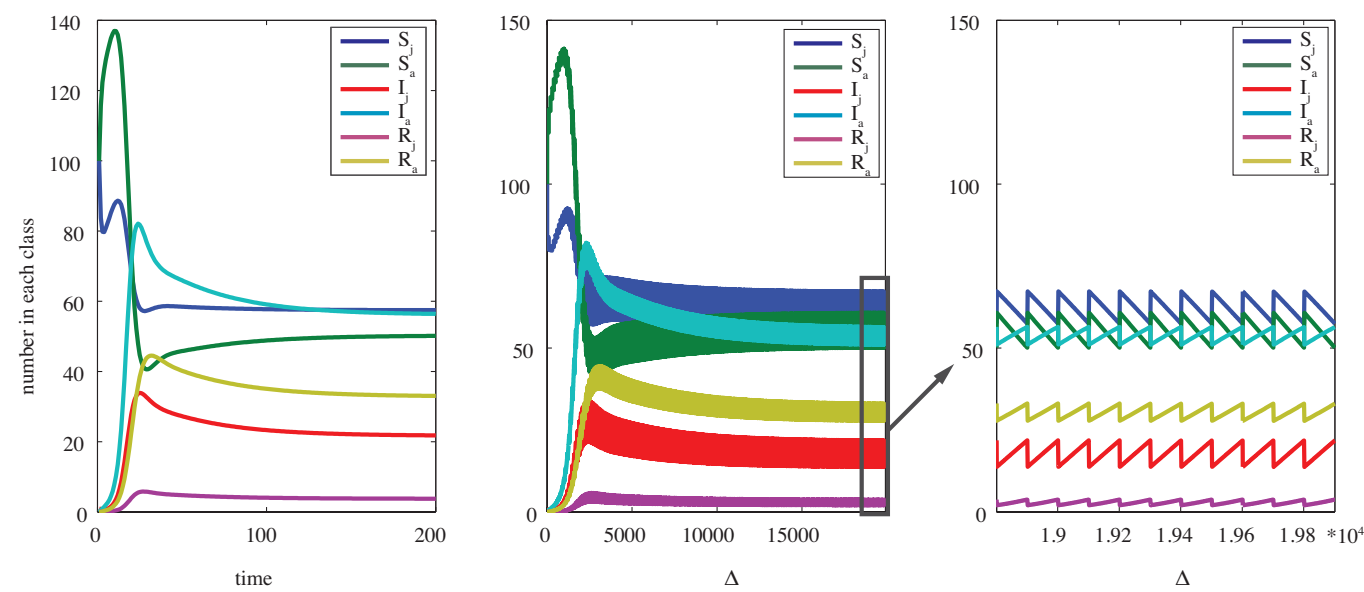

Figure 6: Periodic solution of the periodic stage-structured epidemic model with parameters scaled by time. 
(a)
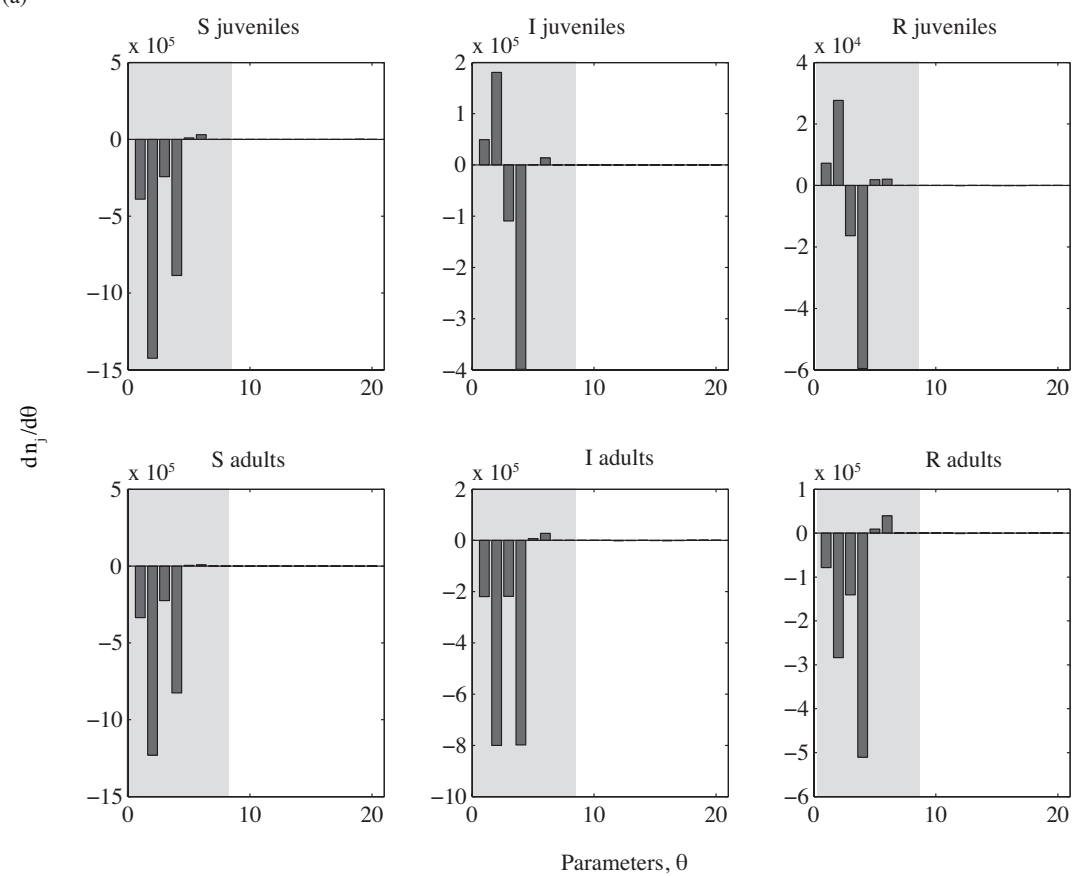

(b)
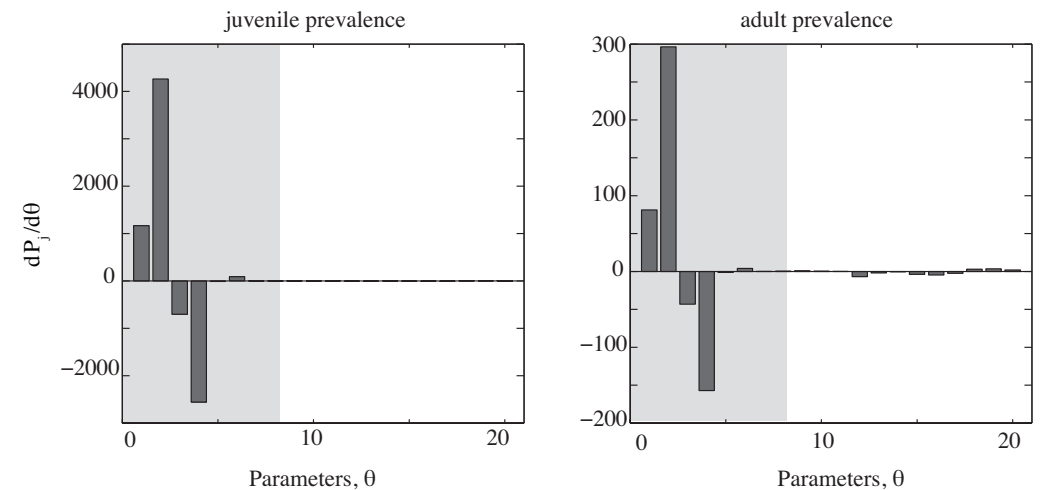

Figure 7: Stage-structured epidemic model with two time scales: (a) sensitivity of the equilibrium population just before the outbreak $\left(\hat{\mathbf{n}}_{1}\right)$; (b) sensitivity of disease prevalence. Parameters as in Figure 4; shaded regions correspond to the epidemic parameters. 
(a)
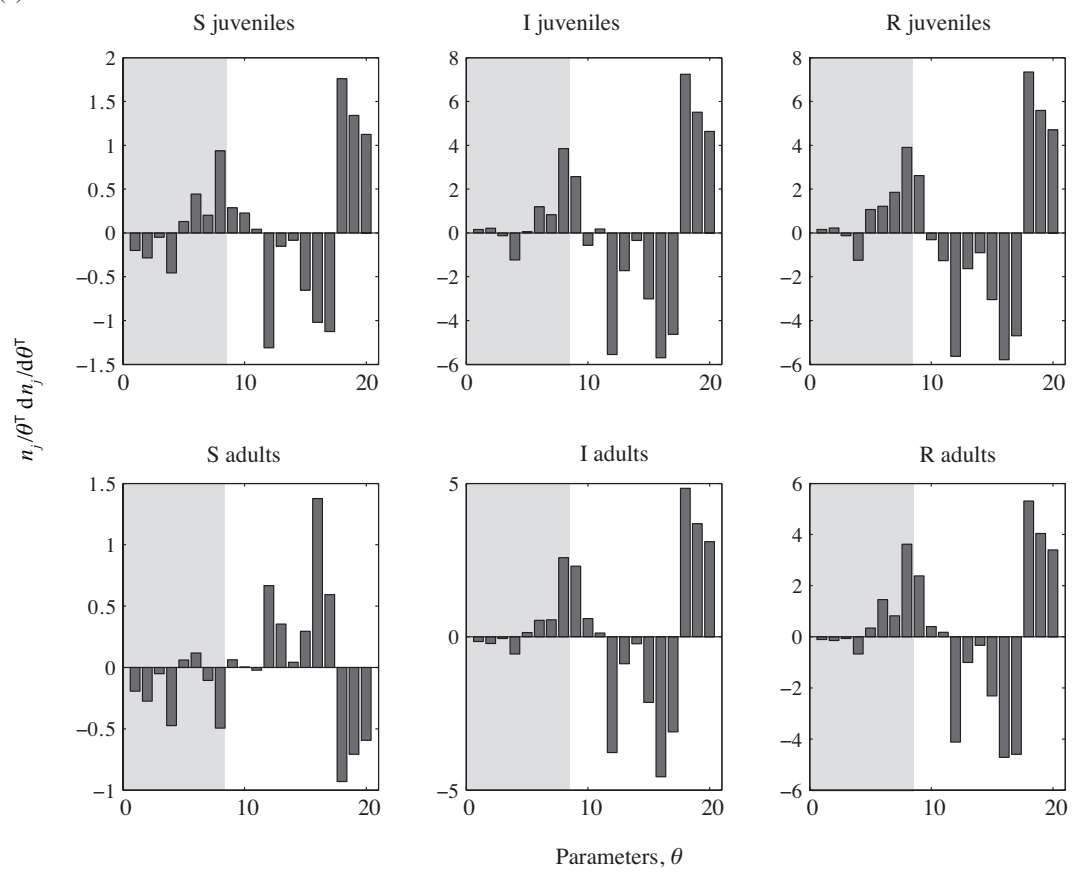

(b)
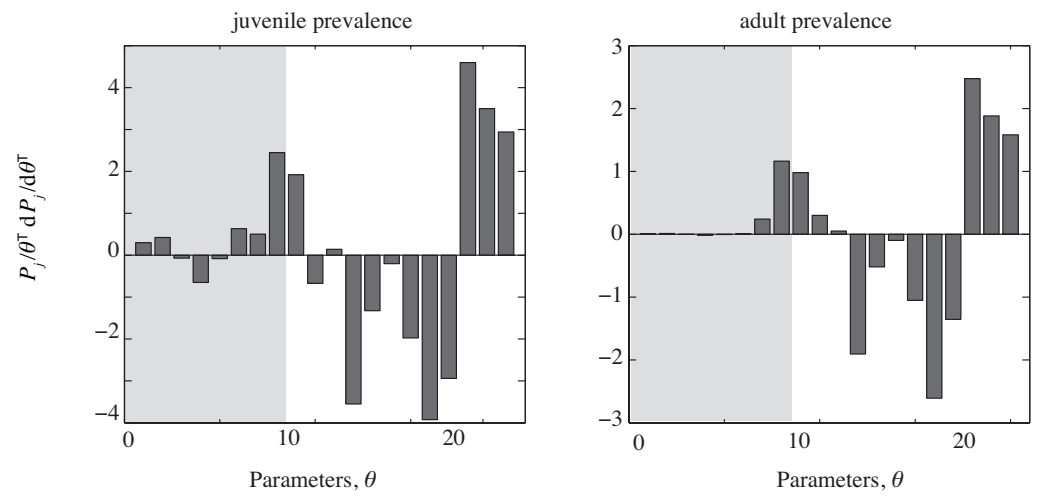

Figure 8: Stage-structured epidemic model with two time scales: (a) elasticities of the equilibrium population just before the outbreak ( $\left.\hat{\mathbf{n}}_{1}\right)$; (b) elasticity of disease prevalence. Parameters as in Figure 4; shaded regions correspond to the epidemic parameters. 